\title{
Single Charge Current in a Normal Mesoscopic Region Attached to Superconductor Leads via a Coupled Poisson Nonequilibrium Green Function Formalism
}

\author{
David Verrilli, ${ }^{1}$ F. P. Marin, ${ }^{1}$ and Rafael Rangel ${ }^{2}$ \\ ${ }^{1}$ Laboratorio de Física Teórica de Sólidos (LFTS), Centro de Física Teórica y Computacional (CEFITEC), \\ Facultad de Ciencias, Universidad Central de Venezuela, A.P. 47586, Caracas 1041-A, Venezuela \\ ${ }^{2}$ Departamento de Física, Universidad Simón Bolívar, A.P. 89000, Caracas 1080-A, Venezuela \\ Correspondence should be addressed to Rafael Rangel; rerangel@usb.ve
}

Received 31 August 2013; Accepted 30 October 2013; Published 9 April 2014

Academic Editors: A. Ovchinnikov and A. Savchuk

Copyright (C) 2014 David Verrilli et al. This is an open access article distributed under the Creative Commons Attribution License, which permits unrestricted use, distribution, and reproduction in any medium, provided the original work is properly cited.

\begin{abstract}
We study the $I-V$ characteristic of mesoscopic systems or quantum dot (QD) attached to a pair of superconducting leads. Interaction effects in the QD are considered through the charging energy of the QD; that is, the treatment of current transport under a voltage bias is performed within a coupled Poisson nonequilibrium Green function (PNEGF) formalism. We derive the expression for the current in full generality but consider only the regime where transport occurs only via a single particle current. We show for this case and for various charging energies values $U_{0}$ and associated capacitances of the QD the effect on the $I$ - $V$ characteristic. Also the influence of the coupling constants on the $I-V$ characteristic is investigated. Our approach puts forward a novel interpretation of experiments in the strong Coulomb regime.
\end{abstract}

\section{Introduction}

The overall shape of the $I-V$ characteristic of a variety of systems (metals, semiconductors, and molecular conductors) in the nanometer scale sandwiched between metallic or superconductors leads has been recently a matter of study (see $[1,2]$ and references therein). In these systems, the energy level discreteness is quite important since level spacing is comparable with other energy scales $[3,4]$. Indeed, the coupling with the bath modifies drastically the properties of an otherwise uncoupled nanometer system in a sharp contrast with similar nonequilibrium macroscopic systems [5-13]. They constitute hybrid systems. Theoretical studies [14-20] as well as experimental measurements have been done by many research groups [1-3] on such systems mostly at low enough temperature with negligible thermal and nonequilibrium fluctuations.

All the systems mentioned above underlay universal common features with the hybrid superconductor quantum dot devices we want to address in this work $[2,4,21,22]$ : (i) broadened energy levels of the quantum dot due to hybridization with the leads; (ii) spatial potential profile. (iii) a charging energy $U_{0}$ due to the potential profile. An insight behind these issues has been highlighted recently $[23,24]$ for molecular dots. The device we study in this work is shown in Figure 1. It constitutes a spin degenerated quantum dot level, which is coupled to a pair of biased superconductors contacts or leads (source and drain). When a source-drain voltage $V_{d}$ is applied, an electric current flows between the leads and across the quantum dot. The biasing defines a non-equilibrium steady state situation. Such situation is coming from the frustration to establish simultaneously an equilibrium configuration with both leads under a given bias. In addition, a gate voltage $V_{g}$ sets the quantum dot spectrum. However, the charge energy can modify it whenever the density of states is significant. In response to the applied voltages, an actual potential develops inside the dot; that is, an effective electrostatic profile potential inside the mesoscopic region exists in such a way, that it couples to both the electronic non-equilibrium state population and the non-equilibrium electric current. That approach, as introduced by Datta [4], links the electrostatic profile to the electronic population of 


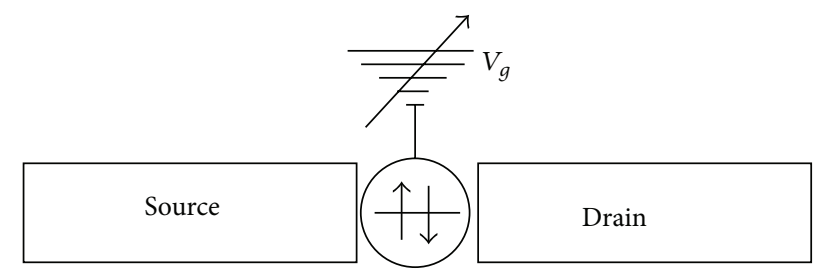

FIGURE 1: Set-up: single level quantum dot connected with two superconducting leads via coupling constants $\Gamma_{s}$.

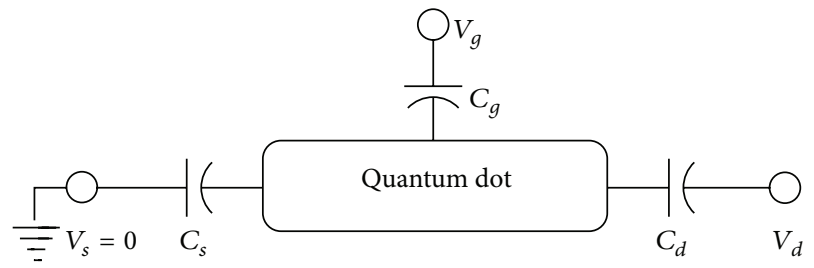

FIGURE 2: Equivalent capacitive circuit with coupling capacitances $C_{s}, C_{g}$, and $C_{d}$, corresponding to the capacitances in the source, gate and drain, respectively.

the quantum dot $[4,25]$ via the non-equilibrium Keldysh formalism (NEGF) $[26,27]$. The whole system is modeled by coupling capacitances which represents the drain, source, and gate contributions to the self-consistent electrostatic problem. Incoming electrons have to overcome an energy barrier (Coulomb blockade). On the other hand, gate or sourcedrain voltage can lower or increase this energy barrier. These source, drain, and gate electrodes capacitances (see Figure 2) constitute a simple capacitive model (in experiments $[2$, 28], these capacitances are measured) from which $U_{\mathscr{L}}$, the Laplacian part of the potential, can be obtained. In addition, the charge in dot can be expressed as the sum of the charges in the coupling capacitances. It yields the Poisson contribution $U_{P}$ to the total potential $U$, as a function of the dot population. In other words, we solve the self-consistency (SC) of the total electrostatic potential $U=U_{\mathscr{L}}+U_{P}$ together with the dot population. After that, the electric current is evaluated.

Previous to the self-consistent program, the non-equilibrium current through the dot and electronic occupation in the dot are worked out. We emphasize that the calculation is carried out in a general framework. However, we confine our attention to the single particle current contribution. We adapt the SC to two different approximation regimes. In Section 4, the equivalent capacitive circuit (Figure 2) is introduced; the spatial potential profile $U$ is calculated within the capacitive model. The SC scheme is applied to two cases $[29,30]$. First, the socalled restricted case, where the gap is the bigger energy scale and the coupling QD-Leads is of the order of the charging energy $\left(\Delta \gg \Gamma_{L, R} \simeq U\right.$ ). In this case, quantitative results are expected to be accurate. We also make calculations for the so-called unrestricted case, where the charging energy is the dominant energy scale $\Delta \simeq U \gg \Gamma_{L, R}$. In this case the results are quantitatively less accurate. The experiments of Ralph et al. [28] were done in this regime. Their $I-V$ characteristic shows that the spacing of the energy levels is subjected to strong fluctuations. According to our model, the fluctuations are due to complex multilevel charging effects. Our hybrid S/QD/S system has been studied in previous theoretical works [16-19]. However, to our knowledge, the coupled SC scheme which describes charging effects has not been considered so far. This is an important step; then, gauge invariant independence of the results as well independence of the zero reference voltage is fulfilled [31,32]. Our model uses experimental values of the equivalent capacitances [4]. To this respect, pioneering work is done by Meir et al. $[33,34]$ for $\mathrm{N} / \mathrm{QD} / \mathrm{N}$ systems, considering the interatomic Coulomb term $U n_{\uparrow} n_{\downarrow}$ as a measure of the charging energy $e^{2} / C$. Their purpose was to find the main object of the non-equilibrium formalism, namely, the QD Green-Keldysh function, in which the influence of the leads on the QD is taken into account. Due to the presence of the Coulomb term, its equation of motion generates a two-particle GreenKeldysh function. By ignoring correlations with the leads, the equation of motion for the QD Green function closes after truncation of higher order equations of motion. This solution (their Equation (8)) has two resonances, one at the energy level weighted by the probability that the other spin degenerate level (raised by $U$ ) is vacant and another one at the energy level raised by $U$ weighted by the probability that the level is occupied. It is correct for temperatures higher than the Kondo temperature and is exact in the noninteracting limit $(U=0)$ and the isolated limit. Analogously, for S/QD/S hybrid systems Kang [16] has obtained an expression for the current through the QD (his Equation (8)), which is evaluated in the $U \rightarrow \infty$ limit (his Equation (13)). The QD Green function from the very beginning does not contain offdiagonal terms that involve superconducting pairing, which excludes the possibility of Andreev reflection processes. The presence in the equation for the current (his Equation (14)) of terms proportional to $\left(1-\left\langle n_{-\sigma}\right\rangle\right)$ affects the contribution to the current of the considered level. In order to complete the outlined program one has to calculate $\left\langle n_{-\sigma}\right\rangle$ self consistently which is not carried out. Instead, Kang calculate the current (his Equation (8)) where the spectral function is calculated in the limit of zero coupling with the leads via a model taken from literature (his Reference [18]) and without taking into account the dependence of the contribution of one level to the current on the occupancy of the other. The point of view which neglects the unavoidable influence of the bath (the leads) on the small system (the QD) is accomplished by factorizing the density matrix $\left(\rho(t)=\rho_{\mathrm{QD}} \otimes \rho_{\text {Baths }}\right)$ and integrating out the leads degrees of freedom which simplify the Liouville-von Neumann equation (Equation (3.140) in [35]). This program is carried out by Kosov et al. for S/QD/S system [36]. In this way, a Markovian master equation is obtained and an expression for the current is calculated. In their Figure 2, they show the $I-V$ characteristic of a nondegenerated QD for a given set of parameters. In this case, the Cooper pair density in the QD is zero [37]. For the sake of comparison, we restrict our calculations to this case. A similar but not identical approach was done by Pfaller et al. [38]. Also, the approach of both Kosov et al. and Pfaller et al. misses the energy levels broadening as discussed in 
the introduction. This lack of broadening is a general deficit of quantum Markov approach [39]. In particular, Pfaller et al. [38] introduce a phenomenological broadening while our approach derives it from first principles. In fact, within the Keldysh formalism, this broadening appears naturally (see (58) below). Levy Yeyati et al. [17] writes an expression for the current (his Equation (2) and Figure 2). They use that expression to explain the experimental results of Ralph et al. [28]. Their calculation was done in the $U \rightarrow \infty$ limit. In addition, they include charging effects, although they do not say explicitly in which way these effects are included. In this respect, one has to realize that $U$ has important contributions to the QD mesoscopic charging effect. In $t \rightarrow-\infty$, the leads and the QD maintain independent thermal equilibrium, that is, are uncoupled systems. When they become coupled, the Keldysh formalism yields the general behavior of the system. After a long enough time, this particular system reaches a steady state.

Our point of view is taken from the fact that the charging of the QD is the origin of the Coulomb repulsion between two electron is occupying a two-fold degenerate level. Therefore, we study the behavior of a noninteracting QD at $t \rightarrow-\infty$ where exact expressions are found. In this way, we obtain a formally similar expression (see (61) below) for the current as Equation (12) in the work of Meir and Wingreen [33]. Later on, Coulomb repulsion is introduced via a self-consistent field (SCF) that depends dynamically on the applied bias $\left(H_{\mathrm{QD}}+U_{\mathrm{SCF}}\right)$ and, in consequence, on the actual number of electrons in the QD. This approach constitutes the coupled Poisson NEGF formalism that has been discussed in the context of molecular conductors by Datta et al. [4, 24]. We use a capacitive model in Section 4 to calculate $U_{\text {SCF }}$ and as discussed above, a numerical procedure is used to evaluate the current. Our approach has the known disadvantage of ignoring correlations in the QD (as pointed out in [39]). In that sense, there is a proposal by Datta (Equation (3.4.9) in [4]) that improves the SCF method and permits more accurate quantitative results. In Section 4, we apply this improvement for the case when the Coulomb charging is greater than the value of the coupling constants. We discuss possible improvements of our approach in Section 6 .

\section{Single Level QD-Model: Derivation of Nonequilibrium Currents}

In macroscopic systems, the task of deriving transport equations or generalized Ginzburg-Landau equations relies on quasiclassical Green functions [7]. In addition, recently non-equilibrium transport in dirty Aluminium quasi-onedimensional nanowires coupled with normal reservoirs [11] was studied experimentally and theoretically with quasiclassical Green functions [13]. As we want to include the possibility of particle interference effects, we do no resort to such objects. This point of view has been discussed in [14]. Instead, we use the equation of motion method (EOM) technique of Keldysh formalism for generating non-equilibrium states (see $[8-10,26])$. We consider a spin degenerated single orbital as a quantum dot connected to superconductors leads.
The Hamiltonian which describes this system is a generalized Anderson model [40]. It reads

$$
H=H_{S}+H_{\mathrm{QD}}+H_{T}
$$

where $H_{S}, H_{\mathrm{QD}}$, and $H_{T}$ stand for the superconducting leads, the dot, and the tunneling term, respectively. Also $H_{S}=$ $\sum_{\eta} H_{\eta}=H_{L}+H_{R}$, where $H_{L}$ and $H_{R}$ are the left and right lead Hamiltonians, respectively. They are given, within the BCS model [41], by

$$
H_{S}=\sum_{\eta \vec{k} \sigma} \Psi_{\eta \vec{k} \sigma}^{\dagger} H_{\eta \vec{k}}^{0} \Psi_{\eta \vec{k} \sigma}
$$

with

$$
H_{\eta \vec{k}}^{0}=\left(\begin{array}{cc}
\varepsilon_{\eta \vec{k}} & \Delta_{\eta \vec{k}} \\
\Delta_{\eta \vec{k}}^{*} & -\varepsilon_{\eta \vec{k}}
\end{array}\right)
$$

where $\varepsilon_{\eta \vec{k}}$ is the conduction electron energy and $\Delta_{\eta \vec{k}}$ is the superconductor gap of the lead $\eta=L, R . \Psi_{\eta \vec{k} \sigma}^{\dagger}$ and $\Psi_{\eta \vec{k} \sigma}$ are the Nambu spinors:

$$
\Psi_{\eta \vec{k} \sigma}^{\dagger}=\left(\begin{array}{cc}
a_{\eta \vec{k} \sigma}^{\dagger} & a_{\eta,-\vec{k},-\sigma}
\end{array}\right), \quad \Psi_{\eta \vec{k} \sigma}=\left(\begin{array}{c}
a_{\eta \vec{k} \sigma} \\
a_{\eta,-\vec{k},-\sigma}^{\dagger}
\end{array}\right) .
$$

Here $a_{\eta \vec{k} \sigma}^{\dagger}\left(a_{\eta \vec{k} \sigma}\right)$ denotes the creation (annihilation) operator for a conduction electron with wave vector $\vec{k}$ and spin $\sigma$ in the $\eta=L, R$ superconductor lead.

$H_{\mathrm{QD}}$ is the hamiltonian for the single-level quantum dot of energy $E_{0}$ :

$$
H_{\mathrm{QD}}=\sum_{\sigma} \phi_{\sigma}^{\dagger} H^{\mathrm{QD}} \phi_{\sigma}
$$

with

$$
H^{\mathrm{QD}}=\left(\begin{array}{cc}
E_{0} & 0 \\
0 & -E_{0}
\end{array}\right) .
$$

The model QD does not contain the Hubbard Coulomb repulsion interaction term. As explained in the introduction, Coulomb repulsion is modeled by means of the inclusion of capacitances, which are taken independent of the charge in the QD. The model also ignores possible superconducting correlations in the QD. For sufficiently small QDs, the discreteness of the single energy levels suppresses these correlations [37]. The position of the energy level will be treated first as fixed by the gate potential with respect to the left lead, while the effect of the applied voltage is taken into account by the coupled Poisson scheme. The tunneling hamiltonian $H_{T}$ is given by

$$
H_{T}=\sum_{\eta \vec{k} \sigma} \Psi_{\eta \vec{k} \sigma}^{\dagger} H_{\eta \vec{k}}^{I} \phi_{\sigma},
$$

with

$$
H_{\eta \vec{k}}^{I}=\left(\begin{array}{cc}
V_{\eta \vec{k}} & 0 \\
0 & -V_{\eta \vec{k}}
\end{array}\right)
$$


$H_{T}$ connects the dot to the biased superconducting leads and it allows the electric charge flow. $V_{\eta \vec{k}}$ is the hybridization matrix element between a conduction electron in the $\eta=L, R$ superconductor lead and a localized electron on the dot with energy $E_{0} . \phi_{\sigma}^{\dagger}$ and $\phi_{\sigma}$ are the dot spinors:

$$
\phi_{\sigma}^{\dagger}=\left(\begin{array}{ll}
d_{\sigma}^{\dagger} & d_{-\sigma}
\end{array}\right), \quad \phi_{\sigma}=\left(\begin{array}{c}
d_{\sigma} \\
d_{-\sigma}^{\dagger}
\end{array}\right)
$$

here, $d_{\sigma}^{\dagger}\left(d_{\sigma}\right)$ is the creation (annihilation) operator for an electron on the dot.

The flow of electric charge from the terminal $\eta$ is given by

$$
I_{\eta}(t)=(-e)\left[-\frac{d\left\langle N_{\eta}(t)\right\rangle}{d t}\right]=\frac{\mathrm{i} e}{\hbar}\left\langle\left[H_{T}(t), N_{\eta}(t)\right]\right\rangle,
$$

where $-e$ is the electron charge. $\langle\cdots\rangle$ is the thermodynamical average over the biased $L$ and $R$ leads at the temperature $T$, taken at time $t_{0} \rightarrow-\infty$, as indicated in the Keldysh contour in Appendix A:

$$
\langle\cdots\rangle \equiv \operatorname{Tr}\left(\rho\left(t_{0}\right) \cdots\right), \quad \rho\left(t_{0}\right) \equiv \frac{e^{-\beta(H-\mu N)}}{\operatorname{Tr}\left(e^{-\beta(H-\mu N)}\right)},
$$

and $N_{\eta}=a_{\eta \vec{k} \sigma}^{\dagger} a_{\eta \vec{k} \sigma}$ is the "number of particles" operator. Book-keeping calculation using (10) leads to

$$
I_{\eta}(t)=\frac{2 e}{\hbar} V_{\eta} \Re \sum_{\vec{k} \sigma} \mathrm{F}_{\eta \vec{k} \sigma}^{<}(t, t) .
$$

$\mathrm{F}_{\eta \vec{k} \sigma}^{<}\left(t, t^{\prime}\right)=\mathrm{i}\left\langle d_{\sigma}^{\dagger}\left(t^{\prime}\right) a_{\eta \vec{k} \sigma}(t)\right\rangle$ is the lesser Keldysh Green function,

$$
\begin{aligned}
\mathrm{F}_{\eta \vec{k} \sigma} & \left(t, t^{\prime}\right) \\
\equiv & -\mathrm{i}\left\langle\mathrm{T}_{\mathrm{K}} a_{\eta \vec{k} \sigma}(t) d_{\sigma}^{\dagger}\left(t^{\prime}\right)\right\rangle \\
\equiv & -\mathrm{i} \Theta\left(t, t^{\prime}\right)\left\langle a_{\eta \vec{k} \sigma}(t) d_{\sigma}^{\dagger}\left(t^{\prime}\right)\right\rangle+\mathrm{i} \Theta\left(t^{\prime}, t\right) \\
& \times\left\langle d_{\sigma}^{\dagger}\left(t^{\prime}\right) a_{\eta \vec{k} \sigma}(t)\right\rangle \\
\equiv & \Theta\left(t, t^{\prime}\right) \mathrm{F}_{\eta \vec{k} \sigma}^{>}\left(t, t^{\prime}\right)+\Theta\left(t^{\prime}, t\right) \mathrm{F}_{\eta \vec{k} \sigma}^{<}\left(t, t^{\prime}\right),
\end{aligned}
$$

and $\mathrm{T}_{\mathrm{K}}$ is the time-ordering operator, the action of which is to rearrange product of operators, such that operator with later times, on the Keldysh contour are placed to the left of the product. Hereafter, for simplicity, we replace $V_{\eta \vec{k}}$ by an average $V_{\eta}$ at the Fermi surfaces $\left(V_{\eta \vec{k}} \equiv \sqrt{\left\langle\left|V_{\eta \vec{k}}\right|^{2}\right\rangle_{\mathrm{FS}}}\right)$ of the leads $L$ and $R$. Using the scheme given in Appendix A for the rate of change of (13), we proceed to obtain the equation of motion:

$$
\begin{aligned}
& \mathrm{i} \frac{\partial \mathrm{F}_{\eta \vec{k} \sigma}\left(t, t^{\prime}\right)}{\partial t} \\
& \quad=\delta\left(t, t^{\prime}\right)\left\langle\left\{a_{\eta \vec{k} \sigma}(t), d_{\sigma}^{\dagger}(t)\right\}\right\rangle-\mathrm{i}\left\langle\mathrm{T}_{\mathrm{K}}\left[a_{\eta \vec{k} \sigma}(t), H\right] d_{\sigma}^{\dagger}\left(t^{\prime}\right)\right\rangle,
\end{aligned}
$$

which leads to

$$
\left(\mathrm{i} \frac{\partial}{\partial t}-\epsilon_{\eta \vec{k}}\right) \mathrm{F}_{\eta \vec{k} \sigma}\left(t, t^{\prime}\right)=-\sigma \Delta_{\eta} \mathscr{F}_{\eta \vec{k} \sigma}\left(t, t^{\prime}\right)+V_{\eta} \mathrm{G}_{\sigma}\left(t, t^{\prime}\right) \text {, }
$$

where

$$
\begin{gathered}
\mathscr{F}_{\eta \vec{k} \sigma}\left(t, t^{\prime}\right)=-\mathrm{i}\left\langle\mathrm{T}_{\mathrm{K}} a_{\eta \vec{k},-\sigma}^{\dagger}(t) d_{\sigma}^{\dagger}\left(t^{\prime}\right)\right\rangle, \\
\mathrm{G}_{\sigma}\left(t, t^{\prime}\right)=-\mathrm{i}\left\langle\mathrm{T}_{\mathrm{K}} d_{\sigma}(t) d_{\sigma}^{\dagger}\left(t^{\prime}\right)\right\rangle .
\end{gathered}
$$

Note that $\mathrm{G}_{\sigma}\left(t, t^{\prime}\right)$ is the QD single-particle Green function. Similarly, $\mathscr{F}_{\eta \vec{k} \sigma}\left(t, t^{\prime}\right)$ satisfies the equation of motion:

$$
\left(\mathrm{i} \frac{\partial}{\partial t}+\epsilon_{\eta \vec{k}}\right) \mathscr{F}_{\eta \vec{k} \sigma}\left(t, t^{\prime}\right)=-\sigma \Delta_{\eta} \mathrm{F}_{\eta \vec{k} \sigma}\left(t, t^{\prime}\right)-V_{\eta} \mathscr{G}_{\sigma}\left(t, t^{\prime}\right),
$$

where

$$
\mathscr{G}_{\sigma}\left(t, t^{\prime}\right)=-\mathrm{i}\left\langle\mathrm{T}_{\mathrm{K}} d_{-\sigma}^{\dagger}(t) d_{\sigma}^{\dagger}\left(t^{\prime}\right)\right\rangle .
$$

Here $\mathscr{G}_{\sigma}\left(t, t^{\prime}\right)$ is the QD of two-particle Green's function.

Equations (15) and (18) can be written in a compact form as follows (see Appendix B):

$$
\begin{aligned}
& \left(\begin{array}{cc}
\mathrm{i} \frac{\partial}{\partial t}-\epsilon_{\eta \vec{k}} & \sigma \Delta_{\eta} \\
\sigma \Delta_{\eta} & \mathrm{i} \frac{\partial}{\partial t}+\epsilon_{\eta \vec{k}}
\end{array}\right)\left(\begin{array}{cc}
\mathrm{F}_{\eta \vec{k} \sigma}\left(t, t^{\prime}\right) & \widetilde{\mathscr{F}}_{\eta \vec{k} \sigma}\left(t, t^{\prime}\right) \\
\mathscr{F}_{\eta \vec{k} \sigma}\left(t, t^{\prime}\right) & \widetilde{\mathrm{F}}_{\eta \vec{k} \sigma}\left(t, t^{\prime}\right)
\end{array}\right) \\
& =V_{\eta} \sigma_{z}\left(\begin{array}{cc}
\mathrm{G}_{\sigma}\left(t, t^{\prime}\right) & \widetilde{\mathscr{G}}_{\eta \vec{k} \sigma}\left(t, t^{\prime}\right) \\
\mathscr{G}_{\sigma}\left(t, t^{\prime}\right) & \widetilde{\mathrm{G}}_{\eta \vec{k} \sigma}\left(t, t^{\prime}\right)
\end{array}\right) .
\end{aligned}
$$

We introduce the tilde Keldysh-Green functions:

$$
\begin{gathered}
\widetilde{\mathscr{F}}_{\eta \vec{k} \sigma}\left(t, t^{\prime}\right)=-\mathrm{i}\left\langle\mathrm{T}_{\mathrm{K}} a_{\eta \vec{k} \sigma}(t) d_{-\sigma}\left(t^{\prime}\right)\right\rangle, \\
\widetilde{\mathrm{F}}_{\eta \vec{k} \sigma}\left(t, t^{\prime}\right)=-\mathrm{i}\left\langle\mathrm{T}_{\mathrm{K}} a_{\eta-\vec{k}-\sigma}^{\dagger}(t) d_{-\sigma}\left(t^{\prime}\right)\right\rangle, \\
\widetilde{\mathscr{G}}_{\sigma}\left(t, t^{\prime}\right)=-\mathrm{i}\left\langle\mathrm{T}_{\mathrm{K}} d_{\sigma}(t) d_{-\sigma}\left(t^{\prime}\right)\right\rangle, \\
\widetilde{\mathrm{G}}_{\sigma}\left(t, t^{\prime}\right)=-\mathrm{i}\left\langle\mathrm{T}_{\mathrm{K}} d_{-\sigma}^{\dagger}(t) d_{-\sigma}\left(t^{\prime}\right)\right\rangle .
\end{gathered}
$$

Consider the following $2 \times 2$ matrix whose elements are the unperturbed Green-Keldysh functions, that is, defined for $V_{\eta}=0$ :

$$
\left(\begin{array}{cc}
\mathrm{g}_{\eta \vec{k} \sigma}\left(t, t^{\prime}\right) & \mathrm{f}_{\eta \vec{k} \sigma}\left(t, t^{\prime}\right) \\
\mathrm{f}_{\eta \vec{k} \sigma} \widetilde{\mathrm{f}}_{\eta \vec{k} \sigma}\left(t, t^{\prime}\right) & \widetilde{\mathrm{g}}_{\eta \vec{k} \sigma}\left(t, t^{\prime}\right)
\end{array}\right)
$$

where

$$
\begin{gathered}
\mathrm{g}_{\eta \vec{k} \sigma}\left(t, t^{\prime}\right) \equiv-\mathrm{i}\left\langle\mathrm{T}_{\mathrm{K}} a_{\eta \vec{k} \sigma}(t) a_{\eta \vec{k} \sigma}^{\dagger}\left(t^{\prime}\right)\right\rangle_{0} \\
\widetilde{\mathrm{f}}_{\eta \vec{k} \sigma}\left(t, t^{\prime}\right) \equiv-\mathrm{i}\left\langle\mathrm{T}_{\mathrm{K}} a_{\eta \vec{k} \sigma}(t) a_{\eta-\vec{k}-\sigma}\left(t^{\prime}\right)\right\rangle_{0} \\
\mathrm{f}_{\eta \vec{k} \sigma}\left(t, t^{\prime}\right) \equiv-\mathrm{i}\left\langle\mathrm{T}_{\mathrm{K}} a_{\eta-\vec{k}-\sigma}^{\dagger}(t) a_{\eta \vec{k} \sigma}^{\dagger}\left(t^{\prime}\right)\right\rangle_{0} \\
\widetilde{\mathrm{g}}_{\eta \vec{k} \sigma}\left(t, t^{\prime}\right) \equiv-\mathrm{i}\left\langle\mathrm{T}_{\mathrm{K}} a_{\eta-\vec{k}-\sigma}^{\dagger}(t) a_{\eta-\vec{k}-\sigma}\left(t^{\prime}\right)\right\rangle_{0} .
\end{gathered}
$$


According to Appendix A, their equations of motion are given by

$$
\begin{gathered}
\left(\mathrm{i} \frac{\partial}{\partial t}-\epsilon_{\eta \vec{k}}\right) \mathrm{g}_{\eta \vec{k} \sigma}\left(t, t^{\prime}\right)+\sigma \Delta_{\eta} \mathrm{f}_{\eta \vec{k} \sigma}\left(t, t^{\prime}\right)=\delta\left(t, t^{\prime}\right), \\
\left(\mathrm{i} \frac{\partial}{\partial t}-\epsilon_{\eta \vec{k}}\right) \widetilde{\mathrm{f}}_{\eta \vec{k} \sigma}\left(t, t^{\prime}\right)+\sigma \Delta_{\eta} \widetilde{\mathrm{g}}_{\eta \vec{k} \sigma}\left(t, t^{\prime}\right)=0, \\
\left(\mathrm{i} \frac{\partial}{\partial t}+\epsilon_{\eta \vec{k}}\right) \mathrm{f}_{\eta \vec{k} \sigma}\left(t, t^{\prime}\right)+\sigma \Delta_{\eta} \mathrm{g}_{\eta \vec{k} \sigma}\left(t, t^{\prime}\right)=0, \\
\left(\mathrm{i} \frac{\partial}{\partial t}+\epsilon_{\eta \vec{k}}\right) \widetilde{\mathrm{g}}_{\eta \vec{k} \sigma}\left(t, t^{\prime}\right)+\sigma \Delta_{\eta} \widetilde{\mathrm{f}}_{\eta \vec{k} \sigma}\left(t, t^{\prime}\right)=\delta\left(t, t^{\prime}\right) .
\end{gathered}
$$

These equations can be written in matrix form as follows:

$$
\begin{gathered}
\left(\begin{array}{cc}
\mathrm{i} \frac{\partial}{\partial t}-\epsilon_{\eta \vec{k}} & \sigma \Delta_{\eta} \\
\sigma \Delta_{\eta} & \mathrm{i} \frac{\partial}{\partial t}+\epsilon_{\eta \vec{k}}
\end{array}\right)\left(\begin{array}{ll}
\mathrm{g}_{\eta \vec{k} \sigma}\left(t, t^{\prime}\right) & \widetilde{\mathrm{f}}_{\eta \vec{k} \sigma}\left(t, t^{\prime}\right) \\
\mathrm{f}_{\eta \vec{k} \sigma}\left(t, t^{\prime}\right) & \widetilde{\mathrm{g}}_{\eta \vec{k} \sigma}\left(t, t^{\prime}\right)
\end{array}\right) \\
=\left(\begin{array}{cc}
\delta\left(t, t^{\prime}\right) & 0 \\
0 & \delta\left(t, t^{\prime}\right)
\end{array}\right) .
\end{gathered}
$$

Equation (20) can be written as an integral along the Keldysh contour $\mathrm{C}_{\mathrm{K}}$ (for an explanation see Appendix B)

$$
\begin{aligned}
& \left(\begin{array}{cc}
\mathrm{F}_{\eta \vec{k} \sigma}\left(t, t^{\prime}\right) & \widetilde{\mathscr{F}}_{\eta \vec{k} \sigma}\left(t, t^{\prime}\right) \\
\mathscr{F}_{\eta \vec{k} \sigma}\left(t, t^{\prime}\right) & \widetilde{\mathrm{F}}_{\eta \vec{k} \sigma}\left(t, t^{\prime}\right)
\end{array}\right) \\
& =\int_{\mathrm{C}_{\mathrm{K}}} \mathrm{d} t^{\prime \prime}\left(\begin{array}{ll}
\mathrm{g}_{\eta \vec{k} \sigma}\left(t, t^{\prime \prime}\right) & \widetilde{\mathrm{f}}_{\eta \vec{k} \sigma}\left(t, t^{\prime \prime}\right) \\
\mathrm{f}_{\eta \vec{k} \sigma}\left(t, t^{\prime \prime}\right) & \widetilde{\mathrm{g}}_{\eta \vec{k} \sigma}\left(t, t^{\prime \prime}\right)
\end{array}\right) \\
& \times V_{\eta} \sigma_{z}\left(\begin{array}{ll}
\mathrm{G}_{\sigma}\left(t^{\prime \prime}, t^{\prime}\right) & \widetilde{\mathscr{G}}_{\sigma}\left(t^{\prime \prime}, t^{\prime}\right) \\
\mathscr{G}_{\sigma}\left(t^{\prime \prime}, t^{\prime}\right) & \widetilde{\mathrm{G}}_{\sigma}\left(t^{\prime \prime}, t^{\prime}\right)
\end{array}\right) .
\end{aligned}
$$

From the last expression one can read for $\mathrm{F}_{\eta \vec{k} \sigma}\left(t, t^{\prime}\right)$ the following equation:

$$
\begin{aligned}
\mathrm{F}_{\eta \vec{k} \sigma}\left(t, t^{\prime}\right) & \\
=V_{\eta} \int_{\mathrm{C}_{\mathrm{K}}} \mathrm{d} t^{\prime \prime} & {\left[\mathrm{g}_{\eta \vec{k} \sigma}\left(t, t^{\prime \prime}\right) \mathrm{G}_{\sigma}\left(t^{\prime \prime}, t^{\prime}\right)\right.} \\
& \left.-\widetilde{\mathrm{f}}_{\eta \vec{k} \sigma}\left(t, t^{\prime \prime}\right) \mathscr{G}_{\sigma}\left(t^{\prime \prime}, t^{\prime}\right)\right] .
\end{aligned}
$$

We now apply the procedure explained in Appendix C; in order to obtain the $\mathrm{F}_{\eta \vec{k} \sigma}\left(t, t^{\prime}\right)$ lesser component, we obtain

$$
\begin{aligned}
& \mathrm{F}_{\eta \vec{k} \sigma}^{<}\left(t, t^{\prime}\right) \\
&=V_{\eta}\left\{\int_{-\infty}^{\infty} \mathrm{d} t^{\prime \prime}\right. {\left[g_{\eta \vec{k} \sigma}^{(\mathrm{r})}\left(t, t^{\prime \prime}\right) \mathrm{G}_{\sigma}^{<}\left(t^{\prime \prime}, t^{\prime}\right)\right.} \\
&\left.-\widetilde{\mathrm{f}}_{\eta \vec{k} \sigma}^{(\mathrm{r})}\left(t, t^{\prime \prime}\right) \mathscr{G}_{\sigma}^{<}\left(t^{\prime \prime}, t^{\prime}\right)\right] \\
&+\int_{-\infty}^{\infty} \mathrm{d} t^{\prime \prime} {\left[\mathrm{g}_{\eta \vec{k} \sigma}^{<}\left(t, t^{\prime \prime}\right) \mathrm{G}_{\sigma}^{(\mathrm{a})}\left(t^{\prime \prime}, t^{\prime}\right)\right.} \\
&\left.\left.-\widetilde{\mathrm{f}}_{\eta \vec{k} \sigma}^{<}\left(t, t^{\prime \prime}\right) \mathscr{G}_{\sigma}^{(\mathrm{a})}\left(t^{\prime \prime}, t^{\prime}\right)\right]\right\} .
\end{aligned}
$$

Furthermore, the superscripts $(<),(>),(\mathrm{r})$, and (a) correspond to lesser, greater, retarded, and advanced Green's functions, respectively.

Therefore, from $(12), I_{\eta}(t)$ can be written as follows:

$$
I_{\eta}(t)=I_{\eta}^{(1)}(t)+I_{\eta}^{(2)}(t),
$$

with

$$
\begin{aligned}
& I_{\eta}^{(1)}(t) \\
& =\frac{2 e}{\hbar} \Re \sum_{\sigma} \int_{-\infty}^{\infty} \mathrm{d} t^{\prime}\left\{\left[V_{\eta}^{2} \sum_{\vec{k}} \mathrm{~g}_{\eta \vec{k} \sigma}^{(\mathrm{r})}\left(t, t^{\prime}\right)\right] \mathrm{G}_{\sigma}^{<}\left(t^{\prime}, t\right)\right. \\
& \left.+\left[V_{\eta}^{2} \sum_{\vec{k}} \mathrm{~g}_{\eta \vec{k} \sigma}^{<}\left(t, t^{\prime}\right)\right] \mathrm{G}_{\sigma}^{(\mathrm{a})}\left(t^{\prime}, t\right)\right\},
\end{aligned}
$$

$$
\begin{aligned}
& I_{\eta}^{(2)}(t) \\
& =-\frac{2 e}{\hbar} \Re \sum_{\sigma} \int_{-\infty}^{\infty} \mathrm{d} t^{\prime}\{[ \\
& \left.V_{\eta}^{2} \sum_{\vec{k}} \widetilde{\mathrm{f}}_{\eta \vec{k} \sigma}^{(\mathrm{r})}\left(t, t^{\prime}\right)\right] \mathscr{G}_{\sigma}^{<}\left(t^{\prime}, t\right) \\
& \left.+\left[V_{\eta}^{2} \sum_{\vec{k}} \widetilde{\mathrm{f}}_{\eta \vec{k} \sigma}^{<}\left(t, t^{\prime}\right)\right] \mathscr{G}_{\sigma}^{(\mathrm{a})}\left(t^{\prime}, t\right)\right\} .
\end{aligned}
$$

When applying the Fourier transformations, (30) and (31) can be expressed as follows:

$$
\begin{aligned}
& I_{\eta}^{(1)}(t)=\frac{2 e}{h} \mathfrak{R} \sum_{\sigma} \int_{-\infty}^{\infty} \mathrm{d} \omega \int_{-\infty}^{\infty} \frac{\mathrm{d} \omega^{\prime}}{2 \pi} \mathrm{e}^{-\mathrm{i}\left(\omega-\omega^{\prime}\right) t} \\
& \quad \times\left[\Sigma_{\eta}^{(\mathrm{r})}(\omega) \mathrm{G}_{\sigma}^{<}\left(\omega, \omega^{\prime}\right)+\Sigma_{\eta}^{<}(\omega) \mathrm{G}_{\sigma}^{(\mathrm{a})}\left(\omega, \omega^{\prime}\right)\right], \\
& I_{\eta}^{(2)}(t) \\
& =-\frac{2 e}{h} \Re \sum_{\sigma}\left\{\mathrm{e}^{-2 \mathrm{i} \mu_{\eta} t} \int_{-\infty}^{\infty} \mathrm{d} \omega\right. \\
& \times \int_{-\infty}^{\infty} \frac{\mathrm{d} \omega^{\prime}}{2 \pi} \mathrm{e}^{-\mathrm{i}\left(\omega-\omega^{\prime}\right) t} \\
& \times\left[\widetilde{\Xi}_{\eta}^{(\mathrm{r})}(\omega) \sigma \mathscr{G}_{\sigma}^{<}\left(\omega, \omega^{\prime}\right)\right. \\
& \left.\left.+\widetilde{\Xi}_{\eta}^{<}(\omega) \sigma \mathscr{G}_{\sigma}^{(\mathrm{a})}\left(\omega, \omega^{\prime}\right)\right]\right\},
\end{aligned}
$$


with

$$
\begin{gathered}
V_{\eta}^{2} \sum_{\vec{k}} \mathrm{~g}_{\eta \vec{k} \sigma}^{(\mathrm{r})}\left(t, t^{\prime}\right) \equiv \int_{-\infty}^{\infty} \frac{\mathrm{d} \omega}{2 \pi} \mathrm{e}^{-\mathrm{i} \omega\left(t-t^{\prime}\right)} \Sigma_{\eta}^{(\mathrm{r})}(\omega), \\
V_{\eta}^{2} \sum_{\vec{k}} \mathrm{~g}_{\eta \vec{k} \sigma}^{<}\left(t, t^{\prime}\right) \equiv \int_{-\infty}^{\infty} \frac{\mathrm{d} \omega}{2 \pi} \mathrm{e}^{-\mathrm{i} \omega\left(t-t^{\prime}\right)} \Sigma_{\eta}^{<}(\omega), \\
V_{\eta}^{2} \sum_{\vec{k}}^{\sim} \widetilde{\mathrm{f}}_{\eta \vec{k} \sigma}^{(\mathrm{r})}\left(t, t^{\prime}\right) \equiv \int_{-\infty}^{\infty} \mathrm{e}^{-2 i \mu_{\eta} t} \sigma \frac{\mathrm{d} \omega}{2 \pi} \mathrm{e}^{-\mathrm{i} \omega\left(t-t^{\prime}\right)} \widetilde{\Xi}_{\eta}^{(\mathrm{r})}(\omega), \\
V_{\eta}^{2} \sum_{\vec{k}}^{\sim \widetilde{f}_{\eta \vec{k} \sigma}}\left(t, t^{\prime}\right) \equiv \int_{-\infty}^{\infty} \mathrm{e}^{-2 i \mu_{\eta} t} \sigma \frac{\mathrm{d} \omega}{2 \pi} \mathrm{e}^{-\mathrm{i} \omega\left(t-t^{\prime}\right)} \widetilde{\Xi}_{\eta}^{<}(\omega) .
\end{gathered}
$$

In Appendices $\mathrm{D}$ to $\mathrm{G}$, we evaluate the unperturbed Green's functions $\mathrm{g}_{\eta \vec{k} \sigma}^{(\mathrm{r})}\left(t, t^{\prime}\right), \quad \mathrm{g}_{\eta \vec{k} \sigma}^{<}\left(t, t^{\prime}\right), \quad \vec{f}_{\eta \vec{k} \sigma}^{(\mathrm{r})}\left(t, t^{\prime}\right)$, and $\widetilde{\mathrm{f}}_{\eta \vec{k} \sigma}\left(t, t^{\prime}\right)$ in the wide band limit.

We summarize these results as follows:

$$
\begin{gathered}
\Sigma_{\eta}^{(\mathrm{r})}(\omega)=-\Gamma_{\eta}\left[\frac{\omega-\mu_{\eta}}{\Delta_{\eta}} \zeta\left(\Delta_{\eta}, \omega-\mu_{\eta}\right)+\mathrm{i} \zeta\left(\omega-\mu_{\eta}, \Delta_{\eta}\right)\right], \\
\Sigma_{\eta}^{<}(\omega)=2 \mathrm{i} \Gamma_{\eta} \zeta\left(\omega-\mu_{\eta}, \Delta_{\eta}\right) \mathrm{f}\left(\omega-\mu_{\eta}\right),
\end{gathered}
$$

$$
\begin{aligned}
\mathrm{i} \frac{\partial}{\partial t} & \left(\begin{array}{cc}
\mathrm{G}_{\sigma}\left(t, t^{\prime}\right) & \widetilde{\mathscr{G}}_{\sigma}\left(t, t^{\prime}\right) \\
\mathscr{G}_{\sigma}\left(t, t^{\prime}\right) & \widetilde{\mathrm{G}}_{\sigma}\left(t, t^{\prime}\right)
\end{array}\right) \\
& =\left(\begin{array}{cc}
\delta\left(t, t^{\prime}\right)-\mathrm{i}\left\langle\mathrm{T}_{\mathrm{K}}\left[d_{\sigma}(t), H\right] d_{\sigma}^{\dagger}\left(t^{\prime}\right)\right\rangle & -\mathrm{i}\left\langle\mathrm{T}_{\mathrm{K}}\left[d_{\sigma}(t), H\right] d_{-\sigma}\left(t^{\prime}\right)\right\rangle \\
-\mathrm{i}\left\langle\mathrm{T}_{\mathrm{K}}\left[d_{-\sigma}^{\dagger}(t), H\right] d_{\sigma}^{\dagger}\left(t^{\prime}\right)\right\rangle & \delta\left(t, t^{\prime}\right)-\mathrm{i}\left\langle\mathrm{T}_{\mathrm{K}}\left[d_{-\sigma}^{\dagger}(t), H\right] d_{-\sigma}\left(t^{\prime}\right)\right\rangle
\end{array}\right),
\end{aligned}
$$

Again using the scheme given in Appendix A, their equation of motion is

$$
\begin{gathered}
\widetilde{\Xi}_{\eta}^{\mathrm{r})}(\omega)=\Gamma_{\eta}\left[\zeta\left(\Delta_{\eta}, \omega+\mu_{\eta}\right)+\mathrm{i} \frac{\Delta_{\eta}}{\omega+\mu_{\eta}} \zeta\left(\omega+\mu_{\eta}, \Delta_{\eta}\right)\right], \\
\widetilde{\Xi}_{\eta}^{<}(\omega)=-2 \mathrm{i} \Gamma_{\eta} \frac{\Delta_{\eta}}{\omega+\mu_{\eta}} \zeta\left(\omega+\mu_{\eta}, \Delta_{\eta}\right) \mathrm{f}\left(\omega+\mu_{\eta}\right), \\
\zeta\left(\omega, \omega^{\prime}\right) \equiv \Theta\left(|\omega|-\left|\omega^{\prime}\right|\right) \frac{|\omega|}{\sqrt{\omega^{2}-\omega^{\prime 2}}} .
\end{gathered}
$$

All these expressions will used below.

\section{QD Green Function}

We need to evaluate the most important objet for calculations, namely, QD Green's functions given by (17) and (19), as well

$$
\begin{gathered}
\widetilde{\mathrm{G}}_{\sigma}\left(t, t^{\prime}\right)=-\mathrm{i}\left\langle\mathrm{T}_{\mathrm{K}} d_{-\sigma}^{\dagger}(t) d_{-\sigma}\left(t^{\prime}\right)\right\rangle, \\
\widetilde{\mathscr{G}}_{\sigma}\left(t, t^{\prime}\right)=-\mathrm{i}\left\langle\mathrm{T}_{\mathrm{K}} d_{\sigma}(t) d_{-\sigma}\left(t^{\prime}\right)\right\rangle .
\end{gathered}
$$
as their respective tilde functions: which develops to

$$
\begin{aligned}
\mathrm{i} \frac{\partial}{\partial t} \mathrm{G}_{\sigma}\left(t, t^{\prime}\right)= & \delta\left(t, t^{\prime}\right)-\mathrm{i} E_{0}\left\langle\mathrm{~T}_{\mathrm{K}} d_{\sigma}(t) d_{\sigma}^{\dagger}\left(t^{\prime}\right)\right\rangle \\
& -\mathrm{i} \sum_{\eta \vec{k}} V_{\eta}\left\langle\mathrm{T}_{\mathrm{K}} a_{\eta \vec{k} \sigma}(t) d_{\sigma}^{\dagger}\left(t^{\prime}\right)\right\rangle \\
= & \delta\left(t, t^{\prime}\right)+E_{0} \mathrm{G}_{\sigma}\left(t, t^{\prime}\right)+\sum_{\eta \vec{k}} V_{\eta} \mathrm{F}_{\eta \vec{k} \sigma}\left(t, t^{\prime}\right), \\
\mathrm{i} \frac{\partial}{\partial t} \mathscr{G}_{\sigma}\left(t, t^{\prime}\right)= & \mathrm{i} E_{0}\left\langle\mathrm{~T}_{\mathrm{K}} d_{-\sigma}^{\dagger}(t) d_{\sigma}^{\dagger}\left(t^{\prime}\right)\right\rangle \\
& +\mathrm{i} \sum_{\eta \vec{k}} V_{\eta}\left\langle\mathrm{T}_{\mathrm{K}} a_{\eta-\vec{k}-\sigma}^{\dagger}(t) d_{\sigma}^{\dagger}\left(t^{\prime}\right)\right\rangle \\
= & -E_{0} \mathscr{G}_{\sigma}\left(t, t^{\prime}\right)-\sum_{\eta \vec{k}} V_{\eta} \mathscr{F}_{\eta \vec{k} \sigma}\left(t, t^{\prime}\right), \\
\mathrm{i} \frac{\partial}{\partial t} \widetilde{\mathscr{G}}_{\sigma}\left(t, t^{\prime}\right)= & -\mathrm{i} E_{0}\left\langle\mathrm{~T}_{\mathrm{K}} d_{\sigma}(t) d_{-\sigma}\left(t^{\prime}\right)\right\rangle \\
& -\mathrm{i} \sum_{\eta \vec{k}} V_{\eta}\left\langle\mathrm{T}_{\mathrm{K}} a_{\eta \vec{k} \sigma}(t) d_{-\sigma}\left(t^{\prime}\right)\right\rangle
\end{aligned}
$$




$$
\begin{aligned}
= & \left(\begin{array}{cc}
\delta\left(t, t^{\prime}\right) & 0 \\
0 & \delta\left(t, t^{\prime}\right)
\end{array}\right) \\
& +\sum_{\eta \vec{k}} V_{\eta} \sigma_{z}\left(\begin{array}{cc}
\mathrm{F}_{\eta \vec{k} \sigma}\left(t, t^{\prime}\right) & \widetilde{\mathscr{F}}_{\eta \vec{k} \sigma}\left(t, t^{\prime}\right) \\
\mathscr{F}_{\eta \vec{k} \sigma}\left(t, t^{\prime}\right) & \widetilde{\mathrm{F}}_{\eta \vec{k} \sigma}\left(t, t^{\prime}\right)
\end{array}\right) .
\end{aligned}
$$

When $V_{\eta}=0$, one has

$$
\begin{gathered}
\left(\begin{array}{cc}
\mathrm{i} \frac{\partial}{\partial t}-E_{0} & 0 \\
0 & \mathrm{i} \frac{\partial}{\partial t}+E_{0}
\end{array}\right)\left(\begin{array}{cc}
\mathrm{G}_{0}\left(t, t^{\prime}\right) & 0 \\
0 & \widetilde{\mathrm{G}}_{0}\left(t, t^{\prime}\right)
\end{array}\right) \\
=\left(\begin{array}{cc}
\delta\left(t, t^{\prime}\right) & 0 \\
0 & \delta\left(t, t^{\prime}\right)
\end{array}\right),
\end{gathered}
$$

with

$$
\begin{gathered}
\mathrm{G}_{0}\left(t, t^{\prime}\right)=-\mathrm{i}\left\langle\mathrm{T}_{\mathrm{K}} d_{\sigma}(t) d_{\sigma}^{\dagger}\left(t^{\prime}\right)\right\rangle_{0}, \\
\widetilde{\mathrm{G}}_{0}\left(t, t^{\prime}\right)=-\mathrm{i}\left\langle\mathrm{T}_{\mathrm{K}} d_{-\sigma}^{\dagger}(t) d_{-\sigma}\left(t^{\prime}\right)\right\rangle_{0}, \\
\left.\mathrm{G}_{0}\left(t, t^{\prime}\right) \equiv \mathrm{G}_{\sigma}\left(t, t^{\prime}\right)\right|_{V_{\eta}=0^{\prime}} \\
\left.\widetilde{\mathrm{G}}_{0}\left(t, t^{\prime}\right) \equiv \widetilde{\mathrm{G}}_{\sigma}\left(t, t^{\prime}\right)\right|_{V_{\eta}=0} .
\end{gathered}
$$

The last two equations can be written as follows:

$$
\begin{aligned}
& \left(\begin{array}{cc}
\mathrm{i} \frac{\partial}{\partial t}-E_{0} & 0 \\
0 & \mathrm{i} \frac{\partial}{\partial t}+E_{0}
\end{array}\right) \\
& \quad \times\left(\begin{array}{cc}
\mathrm{G}_{\sigma}\left(t, t^{\prime}\right)-\mathrm{G}_{0}\left(t, t^{\prime}\right) & \widetilde{\mathscr{G}}_{\sigma}\left(t, t^{\prime}\right) \\
\mathscr{G}_{\sigma}\left(t, t^{\prime}\right) & \widetilde{\mathrm{G}}_{\sigma}\left(t, t^{\prime}\right)-\widetilde{\mathrm{G}}_{0}\left(t, t^{\prime}\right)
\end{array}\right) \\
& =\sum_{\eta \vec{k}} V_{\eta} \sigma_{z}\left(\begin{array}{cc}
\mathrm{F}_{\eta \vec{k} \sigma}\left(t, t^{\prime}\right) & \widetilde{\mathscr{F}}_{\eta \vec{k} \sigma}\left(t, t^{\prime}\right) \\
\mathscr{F}_{\eta \vec{k} \sigma}\left(t, t^{\prime}\right) & \widetilde{\mathrm{F}}_{\eta \vec{k} \sigma}\left(t, t^{\prime}\right)
\end{array}\right) .
\end{aligned}
$$

We write the last equation in its equivalent convolution integral along the Keldysh contour (see Appendix B):

$$
\begin{aligned}
& \left(\begin{array}{cc}
\mathrm{G}_{\sigma}\left(t, t^{\prime}\right)-\mathrm{G}_{0}\left(t, t^{\prime}\right) & \widetilde{\mathscr{G}}_{\sigma}\left(t, t^{\prime}\right) \\
\mathscr{G}_{\sigma}\left(t, t^{\prime}\right) & \widetilde{\mathrm{G}}_{\sigma}\left(t, t^{\prime}\right)-\widetilde{\mathrm{G}}_{0}\left(t, t^{\prime}\right)
\end{array}\right) \\
& =\int_{\mathrm{C}_{\mathrm{K}}} \mathrm{d} t^{\prime \prime}\left(\begin{array}{cc}
\mathrm{G}_{0}\left(t, t^{\prime \prime}\right) & 0 \\
0 & \widetilde{\mathrm{G}}_{0}\left(t, t^{\prime \prime}\right)
\end{array}\right) \\
& \quad \times \sum_{\eta \vec{k}} V_{\eta} \sigma_{z}\left(\begin{array}{cc}
\mathrm{F}_{\eta \vec{k} \sigma}\left(t^{\prime \prime}, t^{\prime}\right) & \widetilde{\mathscr{F}}_{\eta \vec{k} \sigma}\left(t^{\prime \prime}, t^{\prime}\right) \\
\mathscr{F}_{\eta \vec{k} \sigma}\left(t^{\prime \prime}, t^{\prime}\right) & \widetilde{\mathrm{F}}_{\eta \vec{k} \sigma}\left(t^{\prime \prime}, t^{\prime}\right)
\end{array}\right) .
\end{aligned}
$$

An equivalent way to write the last equation (using (25)) as a convolution of $\Sigma_{\sigma}\left(t, t^{\prime}\right)$ and $\mathbf{G}_{\sigma}\left(t, t^{\prime}\right)$ is

$$
\begin{aligned}
& \mathbf{G}_{\sigma}\left(t, t^{\prime}\right) \\
& \quad=\mathbf{G}_{0}\left(t, t^{\prime}\right)+\int_{\mathrm{C}_{\mathrm{K}}} \mathrm{d} t^{\prime \prime} \mathbf{G}_{0}\left(t, t^{\prime}\right) \Sigma_{\sigma}\left(t^{\prime}, t^{\prime \prime}\right) \mathbf{G}_{\sigma}\left(t^{\prime \prime}, t^{\prime}\right),
\end{aligned}
$$

with

$$
\begin{gathered}
\mathbf{G}_{\sigma}\left(t, t^{\prime}\right) \equiv\left(\begin{array}{cc}
\mathrm{G}_{\sigma}\left(t, t^{\prime}\right) & \widetilde{\mathscr{G}}_{\sigma}\left(t, t^{\prime}\right) \\
\mathscr{G}_{\sigma}\left(t, t^{\prime}\right) & \widetilde{\mathrm{G}}_{\sigma}\left(t, t^{\prime}\right)
\end{array}\right), \\
\mathbf{G}_{\sigma}\left(t, t^{\prime}\right) \equiv\left(\begin{array}{cc}
\mathrm{G}_{0}\left(t, t^{\prime}\right) & 0 \\
0 & \widetilde{\mathrm{G}}_{0}\left(t, t^{\prime}\right)
\end{array}\right), \\
\equiv \int_{\mathrm{C}_{\mathrm{K}}} \mathrm{d} t^{\prime \prime}\left(\begin{array}{cc}
V_{\eta}^{2} \sum_{\eta \vec{k}} \mathrm{~g}_{\eta \vec{k} \sigma}\left(t^{\prime \prime}, t^{\prime}\right) & -V_{\eta}^{2} \sum_{\eta \vec{k}} \widetilde{\mathrm{f}}_{\eta \vec{k} \sigma}\left(t^{\prime \prime}, t^{\prime}\right) \\
-V_{\eta}^{2} \sum_{\eta \vec{k}} \mathrm{f}_{\eta \vec{k} \sigma}\left(t^{\prime \prime}, t^{\prime}\right) & V_{\eta}^{2} \sum_{\eta \vec{k}}^{\widetilde{\mathrm{g}}_{\eta \vec{k} \sigma}}\left(t^{\prime \prime}, t^{\prime}\right)
\end{array}\right) .
\end{gathered}
$$

We are interested in two regimes: a first regime in which $U_{0} \sim \Gamma<\Delta$ and the Coulomb blockade effects are neglected because in this case the couplings to the leads are not extremely small and the dot capacitance is large enough, a second regime for $U_{0} \sim \Delta>\Gamma$ where Coulomb blockade effects must be taken into account. For both regimes and from now on, we are interested in the case $\mathrm{eV}>\Delta$, where multiple Andreev reflection [42] processes are strongly suppressed. Therefore only the single particle current (SP) has to be considered $I_{\mathrm{SP}}$. From the above considerations, we have that the Keldysh Green function $\mathscr{G}_{\sigma}(\omega)$, which carries information of the quantum dot two-particle Green's function, can be neglected and all relevant information is contained in $\mathrm{G}_{\sigma}(\omega)$.

The Keldysh Green function becomes spin independent; $\mathrm{G}_{\sigma}(\omega) \equiv \mathrm{G}(\omega)$. Element 11 of (43) is given by

$$
\begin{aligned}
\mathrm{G}\left(t, t^{\prime}\right)= & \mathrm{G}_{0}\left(t, t^{\prime}\right)+\int_{\mathrm{C}_{\mathrm{K}}} \mathrm{d} t^{\prime \prime} \int_{\mathrm{C}_{\mathrm{K}}} \mathrm{d} t^{\prime \prime \prime} \mathrm{G}_{0}\left(t, t^{\prime \prime}\right) \\
& \times \Sigma\left(t^{\prime \prime}, t^{\prime \prime \prime}\right) \mathrm{G}\left(t^{\prime \prime \prime}, t^{\prime}\right) .
\end{aligned}
$$

Again, using the recipe given in Appendix C, we obtain for $\mathrm{G}^{<}\left(t, t^{\prime}\right)$ and $\mathrm{G}^{(\mathrm{a})}\left(t, t^{\prime}\right)$ the following:

$$
\begin{aligned}
\mathrm{G}^{<} & \left(t, t^{\prime}\right) \\
= & \mathrm{G}_{0}^{<}\left(t, t^{\prime}\right) \\
& +\left[\int_{-\infty}^{\infty} \mathrm{d} t^{\prime \prime} \int_{-\infty}^{\infty} \mathrm{d} t^{\prime \prime \prime} \mathrm{G}_{0}^{(\mathrm{r})}\left(t, t^{\prime \prime}\right)\right.
\end{aligned}
$$




$$
\begin{gathered}
\times \Sigma^{(\mathrm{r})}\left(t^{\prime \prime}, t^{\prime \prime \prime}\right) \mathrm{G}^{<}\left(t^{\prime \prime \prime}, t^{\prime}\right) \\
+\mathrm{G}_{0}^{(\mathrm{r})}\left(t, t^{\prime \prime}\right) \Sigma^{<}\left(t^{\prime \prime}, t^{\prime \prime \prime}\right) \\
\times \mathrm{G}^{(\mathrm{a})}\left(t^{\prime \prime \prime}, t^{\prime}\right)+\mathrm{G}_{0}^{<}\left(t, t^{\prime \prime}\right) \\
\left.\times \Sigma^{(\mathrm{a})}\left(t^{\prime \prime}, t^{\prime \prime \prime}\right) \mathrm{G}^{(\mathrm{a})}\left(t^{\prime \prime \prime}, t^{\prime}\right)\right], \\
\mathrm{G}^{(\mathrm{a})}\left(t, t^{\prime}\right) \\
=\mathrm{G}_{0}^{(\mathrm{a})}\left(t, t^{\prime}\right) \\
+\int_{-\infty}^{\infty} \mathrm{d} t^{\prime \prime} \int_{-\infty}^{\infty} \mathrm{d} t^{\prime \prime \prime} \mathrm{G}_{0}^{(\mathrm{a})}\left(t, t^{\prime \prime}\right) \Sigma^{(\mathrm{a})}\left(t^{\prime \prime}, t^{\prime \prime \prime}\right) \mathrm{G}^{(\mathrm{a})}\left(t^{\prime \prime \prime}, t^{\prime}\right) .
\end{gathered}
$$

Taking the Fourier transform of (46) results in a set of algebraic equations:

$$
\begin{aligned}
\mathrm{G}^{<}\left(\omega, \omega^{\prime}\right) \\
=2 \pi \delta\left(\omega-\omega^{\prime}\right) \mathrm{G}_{0}^{<}(\omega)+\mathrm{G}_{0}^{(\mathrm{r})}(\omega) \Sigma^{(\mathrm{r})}(\omega) \mathrm{G}^{<}\left(\omega, \omega^{\prime}\right) \\
\quad+\mathrm{G}_{0}^{(\mathrm{r})}(\omega) \Sigma^{<}(\omega) \mathrm{G}^{(\mathrm{a})}\left(\omega, \omega^{\prime}\right)+\mathrm{G}_{0}^{<}(\omega) \Sigma^{(\mathrm{a})}(\omega) \\
\quad \times \mathrm{G}^{(\mathrm{a})}\left(\omega, \omega^{\prime}\right), \\
\mathrm{G}^{(\mathrm{a})}\left(\omega, \omega^{\prime}\right) \\
=2 \pi \delta\left(\omega-\omega^{\prime}\right) \mathrm{G}_{0}^{(\mathrm{a})}(\omega)+\mathrm{G}_{0}^{(\mathrm{a})}(\omega) \Sigma^{(\mathrm{a})}(\omega) \mathrm{G}^{(\mathrm{a})}\left(\omega, \omega^{\prime}\right) .
\end{aligned}
$$

Dot Keldysh Green's functions $\mathrm{G}_{\sigma}^{<}\left(\omega, \omega^{\prime}\right)$ and $\mathrm{G}_{\sigma}^{(\mathrm{a})}\left(\omega, \omega^{\prime}\right)$ are below straightforward evaluated. In this regime, quantities such as currents are independent of time. Therefore, we have

$$
\begin{aligned}
\mathrm{G}^{<}\left(\omega, \omega^{\prime}\right) & =2 \pi \delta\left(\omega-\omega^{\prime}\right) \mathrm{G}^{<}(\omega), \\
\mathrm{G}^{(\mathrm{a})}\left(\omega, \omega^{\prime}\right) & =2 \pi \delta\left(\omega-\omega^{\prime}\right) \mathrm{G}^{(\mathrm{a})}(\omega) .
\end{aligned}
$$

Therefore (47) result in

$$
\begin{aligned}
& G^{<}(\omega)= G_{0}^{<}(\omega)+G_{0}^{(\mathrm{r})}(\omega) \Sigma^{(\mathrm{r})}(\omega) G^{<}(\omega) \\
&+\mathrm{G}_{0}^{(\mathrm{r})}(\omega) \Sigma^{<}(\omega) G^{(\mathrm{a})}(\omega) \\
&+\mathrm{G}_{0}^{<}(\omega) \Sigma^{(\mathrm{a})}(\omega) \mathrm{G}^{(\mathrm{a})}(\omega), \\
& \mathrm{G}^{(\mathrm{a})}(\omega)=\mathrm{G}_{0}^{(\mathrm{a})}(\omega)+\mathrm{G}_{0}^{(\mathrm{a})}(\omega) \Sigma^{(\mathrm{a})}(\omega) \mathrm{G}^{(\mathrm{a})}(\omega) .
\end{aligned}
$$

Solving (50),

$$
\begin{aligned}
G^{(a)}(\omega) & =\frac{1}{G_{0}^{(a)}(\omega)^{-1}-\Sigma^{(a)}(\omega)}=\frac{1}{\omega-E_{0}-\Sigma^{(\mathrm{a})}(\omega)} \\
& =G^{(\mathrm{r})}(\omega)^{*} .
\end{aligned}
$$

Moreover, we know that

$$
\begin{gathered}
\mathrm{G}_{0}^{<}(\omega) \propto \delta\left(\omega-E_{0}\right), \\
\mathrm{G}_{0}^{(\mathrm{a})}(\omega)=\left(\omega-E_{0}-\mathrm{i} 0^{+}\right)^{-1},
\end{gathered}
$$

Resulting in

$$
\mathrm{G}_{0}^{<}(\omega) \Sigma^{(\mathrm{a})}(\omega) \mathrm{G}^{(\mathrm{a})}(\omega)=-\mathrm{G}_{0}^{<}(\omega) .
$$

Equation (49) is reduced to

$$
\begin{aligned}
G^{<}(\omega)= & G_{0}^{(\mathrm{r})}(\omega) \Sigma^{(\mathrm{r})}(\omega) \mathrm{G}^{<}(\omega)+\mathrm{G}_{0}^{(\mathrm{r})}(\omega) \Sigma^{<}(\omega) \mathrm{G}^{(\mathrm{a})}(\omega), \\
\mathrm{G}^{<}(\omega) & =\frac{\Sigma^{<}(\omega) \mathrm{G}^{(\mathrm{a})}(\omega)}{\mathrm{G}_{0}^{(\mathrm{r})}(\omega)^{-1}-\Sigma^{(\mathrm{r})}(\omega)}=\Sigma^{<}(\omega)\left|G^{(\mathrm{r})}(\omega)\right|^{2} \\
& =\pi \Sigma^{<}(\omega) \frac{-\Im G^{(\mathrm{r})}(\omega) / \pi}{\mathfrak{I}\left(\mathrm{G}^{(\mathrm{r})}(\omega)\right)^{-1}}=\pi \frac{\Sigma^{<}(\omega)}{-\Im \Sigma^{(\mathrm{r})}(\omega)} \rho(\omega) .
\end{aligned}
$$

Here $\rho(\omega)$ is the so-called quantum dot spectral function which is given in terms of the imaginary part (Э) of the retarded Keldysh Green function $\mathrm{G}^{(\mathrm{r})}(\omega)$ :

$$
\begin{aligned}
\rho(\omega) & =-\frac{1}{\pi} \Im G^{(\mathrm{r})}(\omega) \\
& =-\frac{1}{\pi} \frac{\mathfrak{J} \Sigma^{(\mathrm{r})}(\omega)}{\omega-E_{0}-\mathfrak{R} \Sigma^{(\mathrm{r})}(\omega)^{2}+\mathfrak{J} \Sigma^{(\mathrm{r})}(\omega)^{2}} .
\end{aligned}
$$

From (30), the single particle current $\left(I_{\mathrm{SP}}\right)$ results in

$$
\begin{aligned}
& I_{\eta \mathrm{SP}}\left(V, E_{0}\right)=\frac{4 e}{h} \mathfrak{R} \int_{-\infty}^{\infty} \mathrm{d} \omega {\left[\Sigma_{\eta}^{(\mathrm{r})}(\omega) \mathrm{G}^{<}(\omega)\right.} \\
&\left.+\Sigma_{\eta}^{<}(\omega) \mathrm{G}^{(\mathrm{a})}(\omega)\right] .
\end{aligned}
$$

Substituting (51) and (55) in (57),

$$
\begin{aligned}
& I_{\eta \mathrm{SP}}\left(V, E_{0}\right) \\
& =\frac{4 e}{h} \int_{-\infty}^{\infty} \mathrm{d} \omega\left[\pi \mathfrak{\Im} \Sigma_{\eta}^{(\mathrm{r})}(\omega) \frac{\mathfrak{\Im} \Sigma^{<}(\omega)}{\mathfrak{J} \Sigma^{(\mathrm{r})}(\omega)} \rho(\omega)\right. \\
& \left.+\mathfrak{J} \Sigma_{\eta}^{<}(\omega) \mathfrak{J G}(\mathrm{r})(\omega)\right] \\
& =\frac{4 \pi e}{h} \int_{-\infty}^{\infty} \mathrm{d} \omega \rho(\omega)\left[\mathfrak{I} \Sigma_{\eta}^{(\mathrm{r})}(\omega) \frac{\mathfrak{\Im} \Sigma^{<}(\omega)}{\mathfrak{I} \Sigma^{(\mathrm{r})}(\omega)} \rho(\omega)\right. \\
& \left.-\Im \Sigma_{\eta}^{<}(\omega)\right] \\
& =\frac{4 \pi e}{h} \int_{-\infty}^{\infty} \mathrm{d} \omega \frac{\rho(\omega)}{\Gamma(\omega)}\left[\Gamma_{\eta}(\omega) \mathfrak{\Im} \Sigma^{<}(\omega)\right. \\
& \left.-\Gamma(\omega) \Im \Sigma_{\eta}^{<}(\omega)\right]
\end{aligned}
$$

with $\Gamma_{\eta}(\omega)=-\mathfrak{J} \Sigma_{\eta}^{(\mathrm{r})}(\omega)=\Gamma_{\eta} \zeta\left(\omega, \Delta_{\eta}\right)$ and $\Gamma(\omega)=\sum_{\eta} \Gamma_{\eta}(\omega)$. In our regime, $e V>\Delta$; therefore, $\mathfrak{R} \Sigma^{(\mathrm{r})}(\omega)$ in the above equations is zero. We use the expression for $\Sigma^{(\mathrm{r})}(\omega)$ from 
Appendix D and obtain the single particle current $I_{\mathrm{SP}} \equiv$ $\left(I_{R, \mathrm{SP}}-I_{L, \mathrm{SP}}\right) / 2$ :

$$
\begin{aligned}
I_{\mathrm{SP}}\left(V, E_{0}\right)=\frac{8 \pi e}{h} \int_{-\infty}^{\infty} \mathrm{d} \omega \frac{\Gamma_{L}(\omega) \Gamma_{R}(\omega+e V)}{\Gamma_{L}(\omega)+\Gamma_{R}(\omega+e V)} \\
\times \rho(\omega)[\mathrm{f}(\omega)-\mathrm{f}(\omega+e V)] .
\end{aligned}
$$

$-e V=\mu_{L}-\mu_{R}$ corresponds to the applied voltage between the superconductors electrodes with chemical potential $\mu_{\eta}$. In the following, we fix the chemical potential $\mu_{L}=0$ and use $e V$ as a measure of $\mu_{R}$. In addition, the QD energy $E_{0}$ is measured with respect to $\mu_{L}$. On the other hand, the limits of integration are given by the functions $\Gamma_{L}(\omega)$ and $\Gamma_{R}(\omega+e V)$. The extra $2 \pi$ factor arises from the dot Keldysh Green functions. $\rho(\omega)$ and $\Gamma(\omega)$ are given by

$$
\begin{gathered}
\rho(\omega)=\frac{\Gamma(\omega) / \pi}{\left(\omega-E_{0}\right)^{2}+\Gamma^{2}(\omega)}, \\
\Gamma(\omega)=\Gamma_{L}(\omega)+\Gamma_{R}(\omega+e V) .
\end{gathered}
$$

At steady state there is no net flow into or out of the mesoscopic channel or quantum dot which yields a stationary particle number in it. The population number $N$, at the dot, is given by

$$
N=2\left[-\mathrm{iG}^{<}(t, t)\right]=2 \int_{-\infty}^{\infty} \frac{\mathrm{d} \omega}{2 \pi \mathrm{i}} \mathrm{G}^{<}(\omega),
$$

which becomes a weighted average over the $L$ and $R$ contacts:

$$
N=2 \int_{-\infty}^{\infty} \mathrm{d} \omega \rho(\omega)\left[\frac{\Gamma_{L}(\omega)}{\Gamma(\omega)} \mathrm{f}(\omega)+\frac{\Gamma_{R}(\omega+e V)}{\Gamma(\omega)} \mathrm{f}(\omega+e V)\right]
$$

For the N/QD/N case, $\Gamma_{R, L}$ are just constants. This case was studied in the context of the generalized quantum master approach (section IV in [39]). That approach permits the inclusion of broadening in a natural way. They obtained Equations similar to (59)-(63).

\section{Coupled Poisson Nonequilibrium Green Function Scheme: The Capacitive Model}

So far, we are not including the side effects of a potential profile inside the mesoscopic channel. On the one hand, its inclusion takes in order zero or Hartree approximation the electron-electron interaction in the QD. Its inclusion also guarantees current independence from the choice of zero potential [32]. Such potential is induced by the action of source, drain and gate applied voltages. In principle, we have to couple the number of population equations. Equation (62), with electric field $U$. However, since the number of quantum levels in the channel is small and the particle number variation is negligible, the potential profile variation inside the channel is negligible. Then it is appropriate to visualize the channel as an equivalent circuit framework (Figure 2). In this framework, we associate capacitances $C_{d}, C_{s}$, and $C_{g}$ with the drain, source and gate, respectively. Whenever drain, source, and gate bias potentials $V_{d}, V_{s}$, and $V_{g}$, respectively, are present, there is an electrostatic potential $V_{\mathrm{QD}}$ inside the $\mathrm{QD}$, which induces an energy shift of the QD energy level $U=-e\left(V_{\mathrm{QD}}-V_{0}\right), V_{0}$ are channel electrostatic potential before we apply the source and drain biases, respectively.

The electronic populations before and after we apply the biases mentioned above are given by

$$
\begin{gathered}
-e N_{0}=C_{d} V_{0}+C_{s} V_{0}+C_{g} V_{0}, \\
-e N=C_{d}\left(V_{\mathrm{QD}}-V_{d}\right)+C_{s}\left(V_{\mathrm{QD}}-V_{s}\right) \\
+C_{g}\left(V_{\mathrm{QD}}-V_{g}\right),
\end{gathered}
$$

respectively. It leads us to

$$
\begin{aligned}
-e \Delta N \equiv-e\left(N-N_{0}\right)= & C_{E}\left(V_{\mathrm{QD}}-V_{0}\right) \\
& -C_{d} V_{d}-C_{s} V_{s}-C_{g} V_{g},
\end{aligned}
$$

where $C_{E}=C_{d}+C_{s}+C_{g}$. Therefore, the energy shift $U$ is given by

$$
U=U_{\mathscr{L}}+\frac{e^{2}}{C_{E}} \Delta N
$$

where

$$
U_{\mathscr{L}} \equiv \frac{C_{d}}{C_{E}}\left(-e V_{d}\right)+\frac{C_{s}}{C_{E}}\left(-e V_{s}\right)+\frac{C_{g}}{C_{E}}\left(-e V_{g}\right)
$$

In the expression for $U, U_{\mathscr{L}}$ represents a uniform shift for all levels, whereas the second term (the Poisson contribution denoted by $U_{P}$ in the introduction) represents a level of repulsion which is proportional to the averaged occupation of the QD level denoted by $N_{0}$ and proportional to the charging energy $U_{0}=e^{2} / C_{E}$. by

On the other hand, one has $\Delta N$ from (62) and (65) given

$$
\Delta N=2 \int_{-\infty}^{\infty} \frac{\mathrm{d} \omega}{2 \pi \mathrm{i}}\left[\mathrm{G}^{<}(\omega, U)-\mathrm{G}^{<}\left(\omega,-e V_{0}\right)\right]
$$

In the expression for $G^{<}(\omega, U)$ (see (55)), the energy level shifts only $\left(E_{0} \Rightarrow\left(E_{0}+U\right)\right)$ in the expression for the QD spectral function $\rho(\omega-U)$. Equations (66) and (68) are coupled nonlinear equations with unknowns $U$ and $\Delta N$. We solve the coupled equations via an iteration procedure. First we guest a value for $\Delta N$ plug this value in $U$, and then we calculate $\Delta N$ with the following equation:

$$
\begin{aligned}
\Delta N=2 \int_{-\infty}^{\infty} \mathrm{d} & \omega \rho(\omega-U) \\
& \times \frac{\Gamma_{L}(\omega) \mathrm{f}(\omega)+\Gamma_{R}(\omega+e V) \mathrm{f}(\omega+e V)}{\Gamma_{L}(\omega)+\Gamma_{R}(\omega+e V)},
\end{aligned}
$$


and so on until convergence is achieved. With the final value of $U$ obtained for a given bias voltage $V, I_{\mathrm{SP}}$ is calculated via the equation:

$$
\begin{aligned}
I_{\mathrm{SP}}(V, U)=\frac{8 \pi e}{h} \int_{-\infty}^{\infty} \mathrm{d} \omega & \frac{\Gamma_{L}(\omega) \Gamma_{R}(\omega+e V)}{\Gamma_{L}(\omega)+\Gamma_{R}(\omega+e V)} \\
& \times \rho(\omega-U)[\mathrm{f}(\omega)-\mathrm{f}(\omega+e V)] .
\end{aligned}
$$

In summary, the procedure for computing $I$ consists of the following steps. (i) Determine the spectral density. (ii) Specify $V_{g}, V_{d}, V_{s}$, and coupling constants. (iii) Iteratively solve (69) and (66). (iv) Evaluate the current from (70) for the $V_{g}, V_{d}$, and $V_{s}$. Once a converged $U$ has been found, the current is finally evaluated.

The way we consider electron-electron interactions imposes restrictions on the possible values of the charging energy $U_{0}$. For the self-consistent scheme to be valid, we have to assume that $\Delta \gg \Gamma_{L, R} \simeq U_{0}$. However, less precisely quantitative results, although qualitative correct results can be obtained if $\Delta \simeq U_{0} \gg \Gamma_{L, R}$, when the so-called Coulomb Blockade energy dominates over the coupling constants. For this case, we use the improvement of the SCF method discussed in the introduction $[4,29,30]$. The self-consistent generalizes to

$$
\begin{aligned}
& U_{\uparrow}=U_{\mathscr{L}}+\frac{e^{2}}{C_{E}}\left(N_{\downarrow}-N_{0}\right), \\
& U_{\downarrow}=U_{\mathscr{L}}-\frac{e^{2}}{C_{E}}\left(N_{\uparrow}-N_{0}\right),
\end{aligned}
$$

where the up-spin level feels a potential due to the downspin electrons and viceversa. Notice the different signs which reflects the Coulomb repulsion between otherwise degenerate levels:

$$
\begin{array}{r}
N_{\uparrow}=\int_{-\infty}^{\infty} \mathrm{d} \omega \rho\left(\omega-U_{\uparrow}\right) \\
\quad \times \frac{\Gamma_{L}(\omega) \mathrm{f}(\omega)+\Gamma_{R}(\omega+e V) \mathrm{f}(\omega+e V)}{\Gamma_{L}(\omega)+\Gamma_{R}(\omega+e V)}, \\
N_{\downarrow}=\int_{-\infty}^{\infty} \mathrm{d} \omega \rho\left(\omega-U_{\downarrow}\right) \\
\quad \times \frac{\Gamma_{L}(\omega) \mathrm{f}(\omega)+\Gamma_{R}(\omega+e V) \mathrm{f}(\omega+e V)}{\Gamma_{L}(\omega)+\Gamma_{R}(\omega+e V)}, \\
N=N_{\uparrow}+N_{\downarrow} .
\end{array}
$$

Here, $N_{\uparrow}$ and $N_{\downarrow}$ are the population of the spin-up and spindown levels. Once the values of $U_{\uparrow}$ and $U_{\downarrow}$ are calculated, $I_{\mathrm{SP}}$ is calculated from

$$
\begin{aligned}
I_{\mathrm{SP}}\left(V, U_{\uparrow}, U_{\downarrow}\right) & \\
=\frac{4 \pi e}{h} \int_{-\infty}^{\infty} & \mathrm{d} \omega \frac{\Gamma_{L}(\omega) \Gamma_{R}(\omega+e V)}{\Gamma_{L}(\omega)+\Gamma_{R}(\omega+e V)} \\
\times & \left\{\rho\left(\omega-U_{\uparrow}\right)+\rho\left(\omega-U_{\downarrow}\right)\right\} \\
\times & {[\mathrm{f}(\omega)-\mathrm{f}(\omega+e V)] . }
\end{aligned}
$$

As Datta has pointed out [4], the approach described above (called unrestricted SCF) can lead to a better quantitative agreement in comparison with a conceptually correct multilevel Master equation calculation.

\section{Numerical Results and Remarks on Experiments}

5.1. First Case: $\Delta \gg \Gamma_{L, R} \simeq U_{0}$. In this regime, there are the multiple Andreev reflections [42] for voltages such that $\mathrm{eV}<$ $\Delta$ (MAR). Also, there is the possibility for quasiparticle cotunneling current for energy levels far from $\mu_{L}$. These cases will be considered in a future work that involves the whole expressions we have derived for the currents (see (30) and (31)) and eventually more accurate Green-Keldysh functions and the use of master equations [17]. This case was studied experimentally in [43]. For given values of capacitances and source voltage, we iterate (66) and (68) in order to find the potential $U$. Then the single particle current $I_{\mathrm{SP}}(V, U)$ is evaluated (see (70)). We put the charge before biasing $N_{0}=$ 0 , such that Coulomb repulsion with the QD-energy level is absent. Anyhow, in this regime, the effect of the second term in (74) is negligible. Consequently, the Laplace term $U_{\mathscr{L}}$ essentially positions the QD degenerate energy level (with respect to $\mu_{L}=0$ ). In Figure 3, we show $I-V$ characteristic for gate voltage values $V_{g}=0$ and $K_{B} T=\Delta$, whereas Figure 4 shows the occupation number $\Delta N$. These curves are symmetric, due to the assumed equality of the coupling capacitances $\left(C_{d} / C_{E}=0.5\right)$. Otherwise, the $I-V$ shifts to right or to the left for $C_{d} / C_{E}=0.5>0.5$ or $<0.5$ respectively. For this case, we show in Figure 7 the spectral density $\rho(\omega)$ for $e V_{d}=-6 \Delta$. Notice that the position of the energy level is essentially $-4.5 \Delta$, that is, just the sum of $E_{0}+U_{\mathscr{L}}$. Qualitatively, these results are similar to Levy Yeyati et al.s [17]. Characteristic is the broadening of the BSC singularity. The effect of bigger values of $\Gamma_{R, L}$ is a more pronounced round off the BCS-type singularity. We discuss this issue below. For large enough bias, the current approaches the normal saturation value $\mathrm{I}_{\text {Sat }}$.

5.2. Second Case: $\Delta \simeq U_{0} \gg \Gamma_{L, R}$. In this regime, the charging energy acts effectively in lifting the degeneracy of the otherwise single degenerate QD energy level. For this regime, we use the couple system defined by (71)(75) and calculate the current according to (76). This is the unrestricted SCF method mentioned in the introduction. 


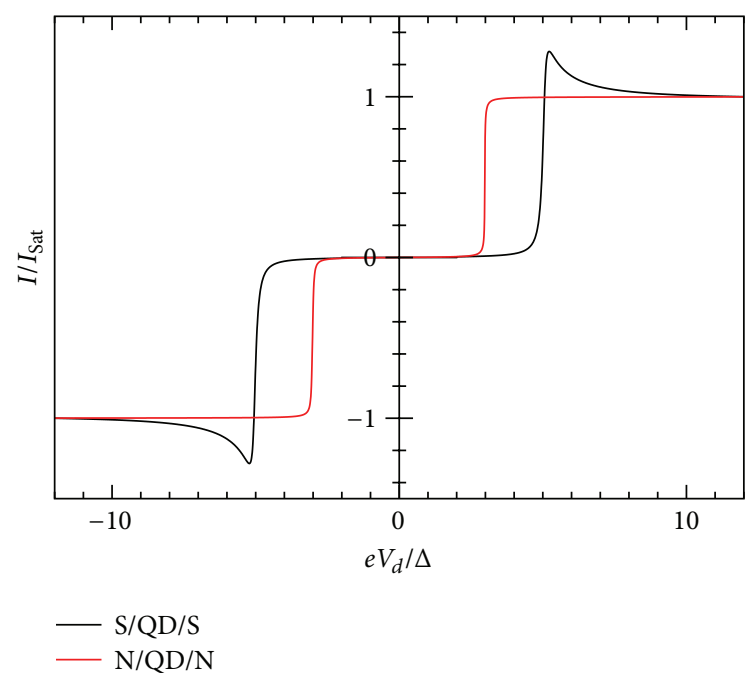

FIgURE 3: Zero temperature $I-V$ characteristics for superconductorquantum-dot-superconductor system, calculated using the selfconsistent field (SCF) method, with $E_{0}=1.5 \Delta, e V_{g}=0.0 \Delta, U_{0}=$ $0.005 \Delta, C_{d} / C_{E}=0.5$, and $\Gamma_{L}=\Gamma_{R}=0.005 \Delta$.

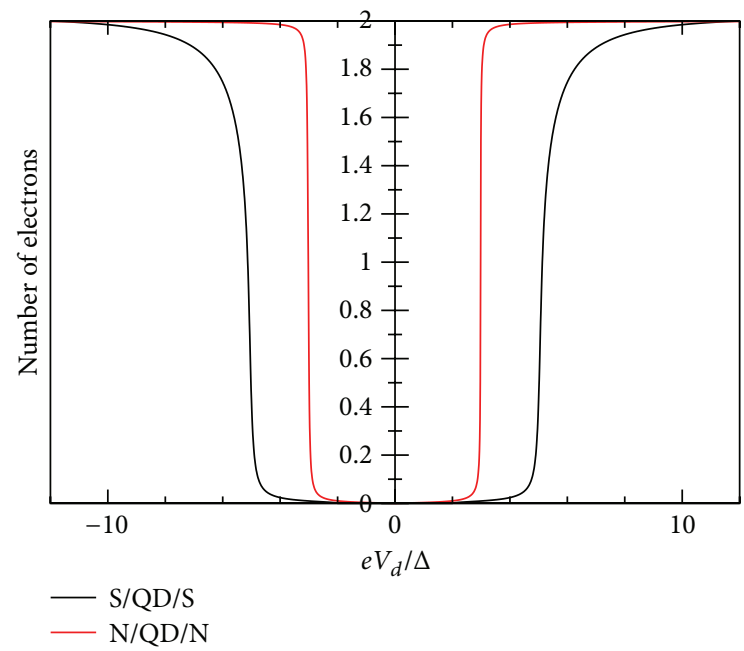

FIGURE 4: Zero temperature number of electrons $e V_{d} / \Delta$ graph for superconductor-quantum-dot-superconductor system, calculated using the self-consistent field (SCF) method, with $E_{0}=1.5 \Delta, e V_{g}=$ $0.0 \Delta, U_{0}=0.005 \Delta, C_{d} / C_{E}=0.5$, and $\Gamma_{L}=\Gamma_{R}=0.005 \Delta$.

The transport begins through one level as long as there is in average less than one electron in it. For the given parameters, the onset of current is similar to the first case (no interaction with residual charge in the QD is considered). However, when the average occupation exceeds one, the other degenerate levels float according to the resulting values $U_{\uparrow}$ and $U_{\downarrow}$. These values push down the position of this second level and push up the already occupied energy level. In Figures 5-6, we show $I-V$ and the number of electrons. In Figure 8, it is shown that the spectral density for $e V_{d}=-8 \Delta$. In this case, $E_{0}+U_{\mathscr{L}}=$ $-5.5 \Delta$. The values obtained from the SCF calculation position the energy levels to $-4.846 \Delta$ and to $-6.396 \Delta$ (see (72)).

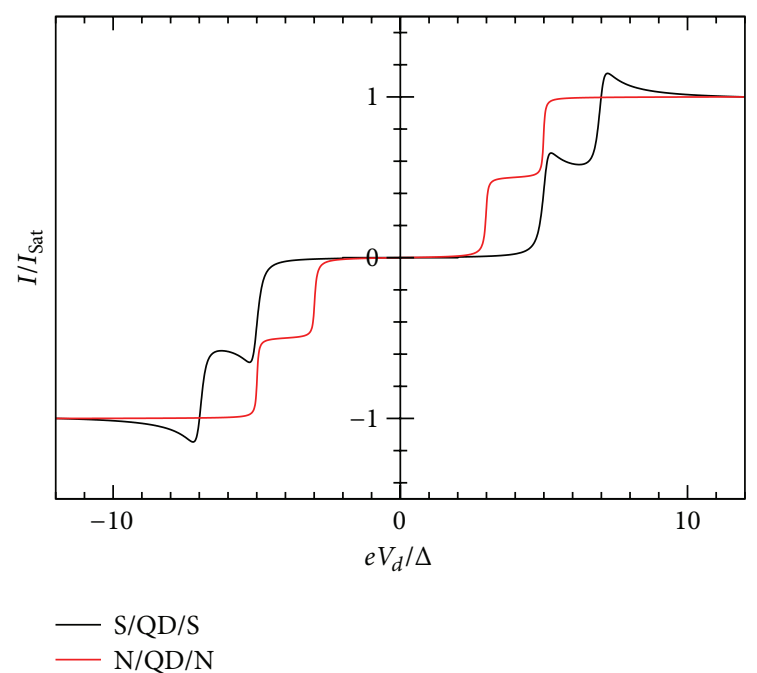

FIGURE 5: Zero temperature $I-V$ characteristic showing the Coulomb blockade for superconductor-quantum-dot-superconductor system, calculated using the self-consistent field (SCF) method, with $E_{0}=1.5 \Delta, e V_{g}=0.0 \Delta, U_{0}=1.0 \Delta, C_{d} / C_{E}=0.5$, and $\Gamma_{L}=\Gamma_{R}=$ $0.01 \Delta$.

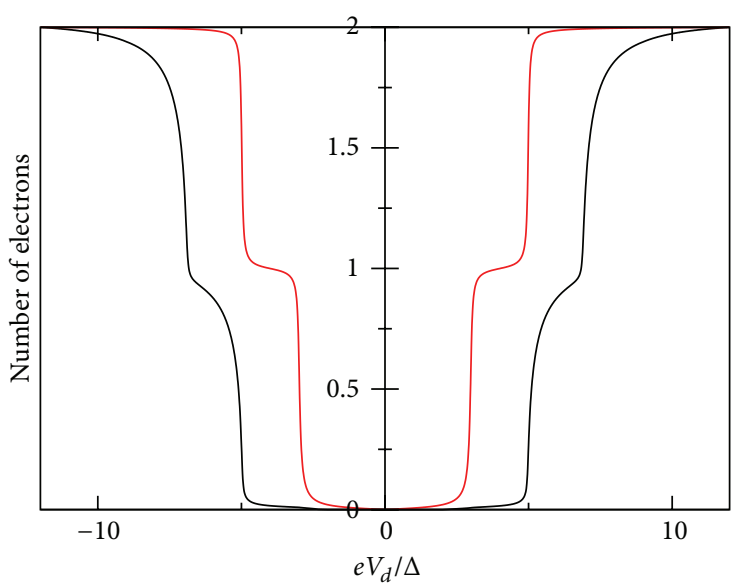

Figure 6: Zero temperature number of electrons- $e V_{d} / \Delta$ for superconductor-quantum-dot-superconductor system, calculated using the self-consistent field (SCF) method, with $E_{0}=1.5 \Delta, e V_{g}=0.0 \Delta$, $U_{0}=1.0 \Delta, C_{d} / C_{E}=0.5$, and $\Gamma_{L}=\Gamma_{R}=0.01 \Delta$.

The experimental work of Ralph et al. [28] corresponds to this second case. In their Figure 3, they show the current for single state level peaks $I \sim 5 \mathrm{pA}$ at a bias $V \sim 2.4 \mathrm{mV}$. According to Figures 5-6, for this sample at $30 \mathrm{mK}$, there should be another peak at $V \sim 4.4 \mathrm{mV}$ with a current value $I_{\mathrm{SP}} \simeq 10 \mathrm{pA}$. The charging energy for this sample is $U_{0} \simeq$ $2.0 \mathrm{mV}$. However, in their samples with radios $\sim 2.5 \mathrm{~nm}$ or greater, the level spacing of the energy levels is such, that $\Delta E<E_{c}$; therefore another current signal may occur before and a quantitative proper description would be a multilevel QD-model. One notice, however, the strong fluctuations in the spacing in Figure 2 [28], indicating complex charging for many levels of phenomena. Notice that the theoretical 


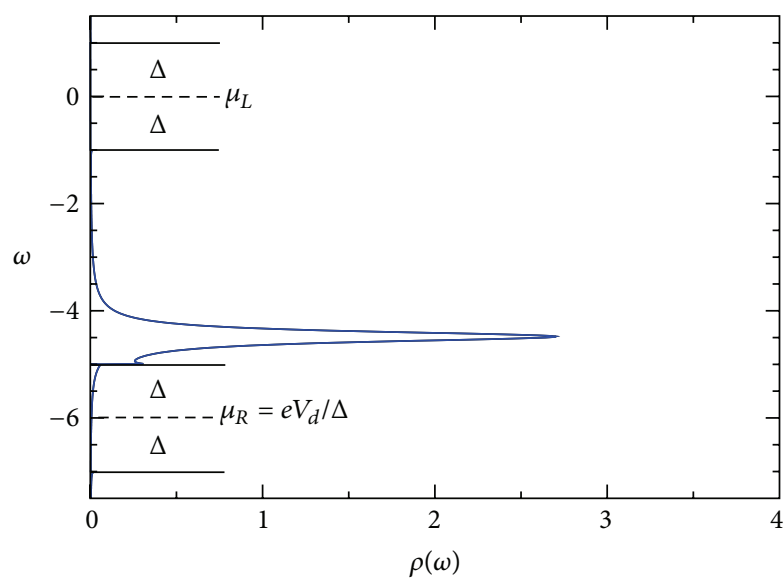

Figure 7: Spectral density of the quantum dot- $\omega$ for superconductor-quantum-dot-superconductor system, calculated using the selfconsistent field (SCF) method, with $E_{0}=1.5 \Delta, e V_{g}=0.0 \Delta, e V_{d}=$ $6.0 \Delta, U_{0}=0.01 \Delta, C_{d} / C_{E}=0.5$, and $\Gamma_{L}=\Gamma_{R}=0.05 \Delta$.

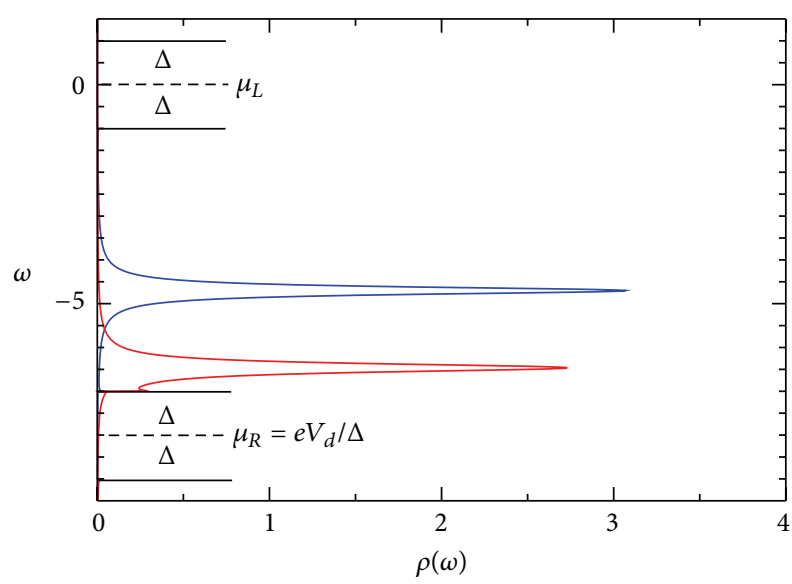

Figure 8: Spectral density of the quantum dot- $\omega$ for superconductor-quantum-dot-superconductor system, calculated using the selfconsistent field (SCF) method, with $E_{0}=1.5 \Delta, \mathrm{eV}_{g}=0.0 \Delta, \mathrm{eV}_{d}=$ $8.0 \Delta, U_{0}=1.0 \Delta, C_{d} / C_{E}=0.5$, and $\Gamma_{L}=\Gamma_{R}=0.20 \Delta$.

explanation of Levy Yeyati et al. $[17,18]$ does not contain our prediction.

5.3. Influence of Coupling Constants. The influence of the coupling constant is to broaden the otherwise sharp energy QD level. However, the broadening is not equally strong and depends on the relative values of $\Gamma_{R} / \Gamma_{L}$. Ralph et al. [28] determined for the sample in his Figure $3 \Gamma_{R} / \Gamma_{L} \gg 1$. Figure 10 shows the $I-V$ characteristic for the restricted case when both coupling constants are equal. For larger values of the coupling constants, the broadening is stronger. If they are dissimilar in value, the broadening is stronger when $\Gamma_{R}>\Gamma_{L}$. This effect is shown in Figures 9, 10,11, 12, 13, and 14. This effect is due to the stronger involving of the BSC-DOS singularity of the left lead in the integral expression for the current (see (59)) and the particular choice of the zero bias voltage (see Figure 2).

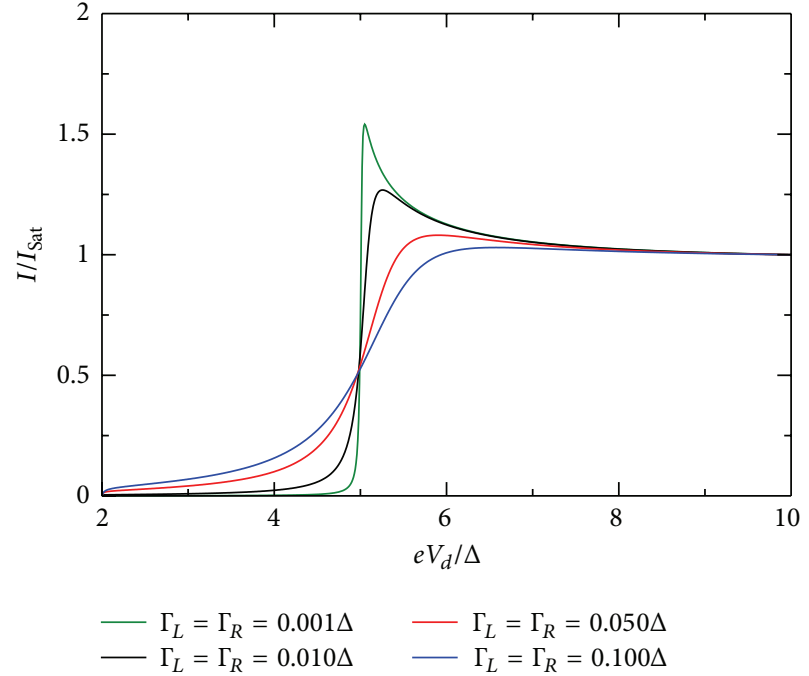

FIGURE 9: Zero temperature $I-V$ characteristic for superconductorquantum-dot-superconductor system for various values of the coupling $\Gamma$, calculated using the self-consistent field (SCF) method, with $E_{0}=1.5 \Delta, e V_{g}=0.0 \Delta, U_{0}=0.05 \Delta, C_{d} / C_{E}=0.5$, and $\Gamma_{L}=\Gamma_{R}$.

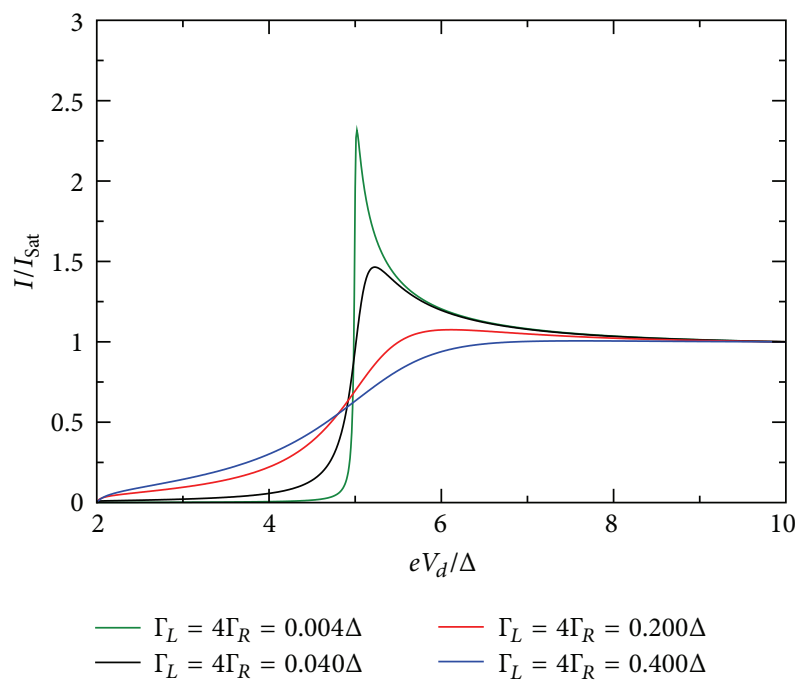

FIgURE 10: Zero temperature $I-V$ characteristic for superconductorquantum-dot-superconductor system for various values of the coupling $\Gamma$, calculated using the self-consistent field (SCF) method, with $E_{0}=1.5 \Delta, e V_{g}=0.0 \Delta, U_{0}=0.05 \Delta, C_{d} / C_{E}=0.5$, and $\Gamma_{L}=4 \Gamma_{R}$.

\section{Summary and Perspectives}

We have studied the single particle current through a quantum dot coupled with two superconductor leads via a coupled Poisson Nonequilibrium Green function (PNEGF) formalism. In a systematic and self-contained way, we derived the expressions for the current in full generality. In this work we focused only on the weak coupling regime where single particle current is the dominant one. The QD is a single degenerate energy level system modeled via a capacitive circuit. The influence of the potential on the QD and on the $I-V$ 


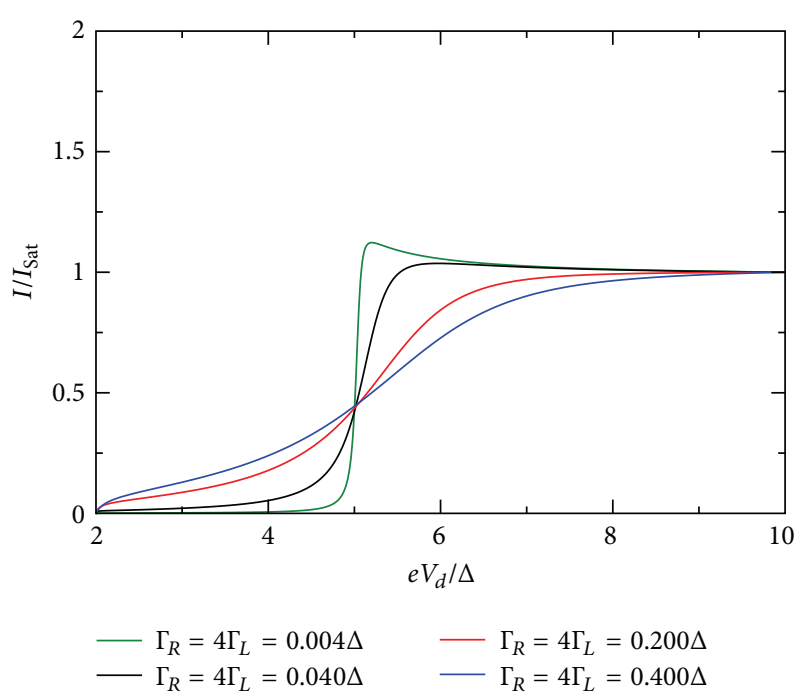

FIGURE 11: Zero temperature $I-V$ characteristic for superconductorquantum-dot-superconductor system for various values of the coupling $\Gamma$, calculated using the self-consistent field (SCF) method, with $E_{0}=1.5 \Delta, e V_{g}=0.0 \Delta, U_{0}=0.05 \Delta, C_{d} / C_{E}=0.5$, and $\Gamma_{R}=4 \Gamma_{L}$.

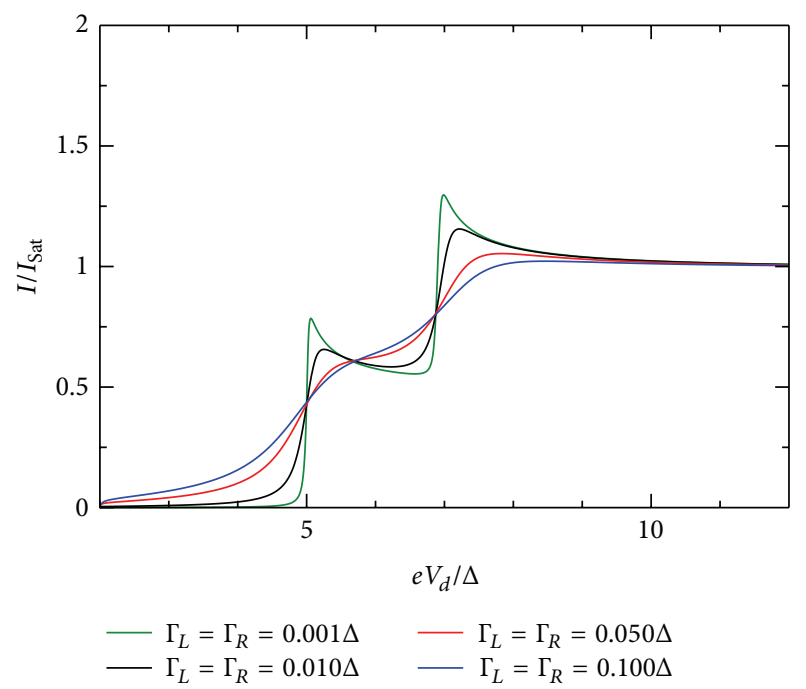

FIGURE 12: Zero temperature $I-V$ characteristic for superconductorquantum-dot-superconductor system for various values of the coupling $\Gamma$, calculated using the self-consistent field (SCF) method, with $E_{0}=1.5 \Delta, e V_{g}=0.0 \Delta, U_{0}=1.0 \Delta, C_{d} / C_{E}=0.5$, and $\Gamma_{L}=\Gamma_{R}$.

characteristic is calculated for relevance values of the coupling and capacitances and the implication of experiments is discussed. This was done in the weak coupling regime and for $\Delta \gg \Gamma_{L, R} \simeq U_{0}$. A second case when $\Delta \simeq U_{0} \gg \Gamma_{L, R}$ also in the weak coupling regime was analyzed. Admittedly, our model of a hybrid system S/QD/S possesses potentially physical extensions. One important missed point is dephasing. This physical effect due to scattering of transport electrons can be incorporated in the self energy phenomenologically $[44,45]$, or in a stochastic fashion [46]. Another point is to consider a QD with many energy levels and within the self-consistent

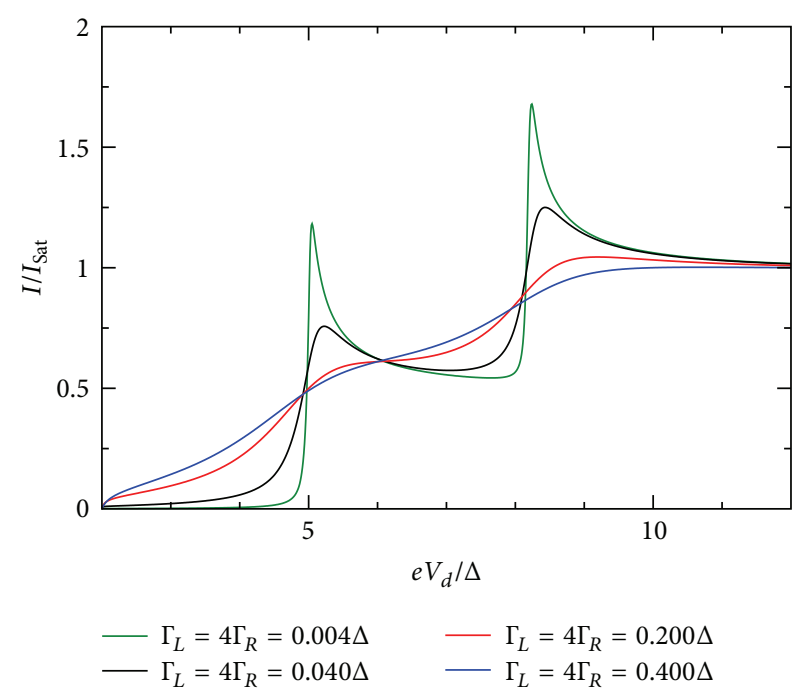

FIGURE 13: Zero temperature $I-V$ characteristic for superconductorquantum-dot-superconductor system for various values of the coupling $\Gamma$, calculated using the self-consistent field (SCF) method, with $E_{0}=1.5 \Delta, e V_{g}=0.0 \Delta, U_{0}=1.0 \Delta, C_{d} / C_{E}=0.5$, and $\Gamma_{L}=4 \Gamma_{R}$.

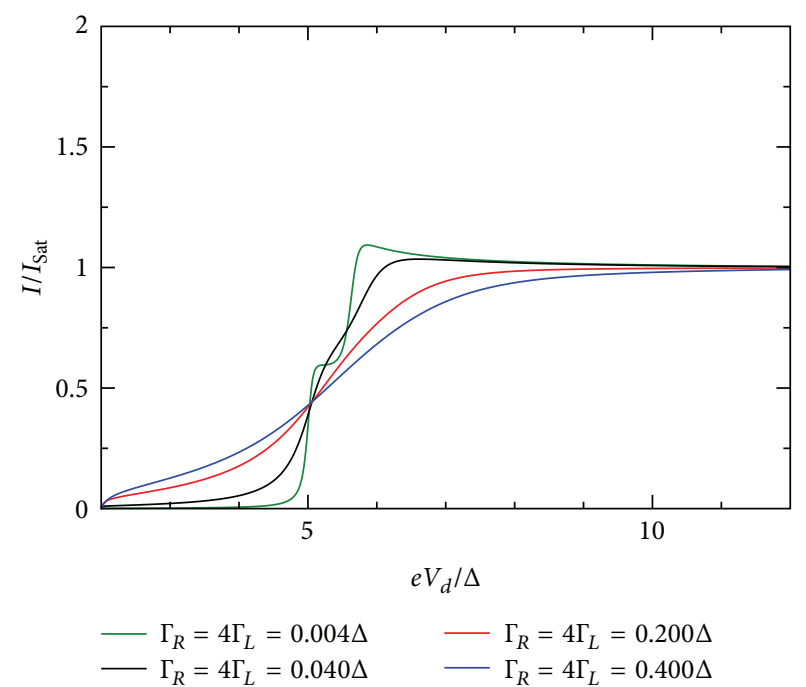

FIGURE 14: Zero temperature $I-V$ characteristic for superconductorquantum-dot-superconductor system for various values of the coupling $\Gamma$, calculated using the self-consistent field (SCF) method, with $E_{0}=1.5 \Delta, e V_{g}=0.0 \Delta, U_{0}=1.0 \Delta, C_{d} / C_{E}=0.5$, and $\Gamma_{R}=4 \Gamma_{L}$.

scheme, to consider the strong and intermediate regimes and many body correlations due to different kinds of electronelectron interaction. Here we have to notice that it is not just to scale the level spacing by the charging energy [47]. It is a genuine many body problem. But the most important missed point was correlations. As pointed out by Datta $[4$, chapter III], there has been much effort in order to find a suitable SCF that considers correlations. For example, to modify (66) to consider occupancies probabilities. As discussed in the introduction, Kang [16] and Meir and Wingreen [33] find a solution for the QD Green function that contains this 
type of correlation. In other words, one could go to scheme where a more accurate Green function for the QD is used together with a multielectron picture and corresponding master equation. These would mean to use the Anderson model with a Coulomb interaction $U$ that is obtained from a SCF. We want to check if this point of view is correct. Work in this direction is in progress.

\section{Appendices}

\section{A. Equation of Motion for the Keldysh-Green Function}

In general, any Green-Keldysh function of two operators $A(t)$ and $B(t)$ function is given by

$$
\mathrm{G}_{A, B}\left(t, t^{\prime}\right)=-\mathrm{i}\left\langle\mathrm{T}_{\mathrm{K}} A(t) B\left(t^{\prime}\right)\right\rangle,
$$

where the operator $T_{K}$ acts on the Keldysh contour shown in Figure 15. A Heaviside function on the Keldysh contour is given by

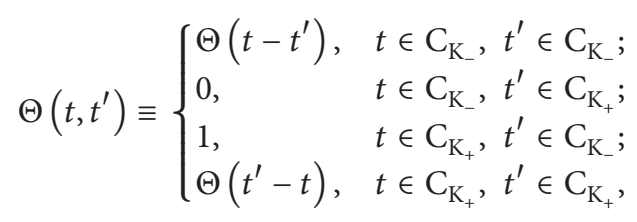

whereas be derivative of the Heaviside function on the Keldysh contour is given by

$$
\begin{aligned}
& \delta\left(t, t^{\prime}\right) \equiv \frac{\partial \Theta\left(t, t^{\prime}\right)}{\partial t}=-\frac{\partial \Theta\left(t, t^{\prime}\right)}{\partial t^{\prime}} \\
& = \begin{cases}\delta\left(t-t^{\prime}\right), & t \in \mathrm{C}_{\mathrm{K}_{-}}, t^{\prime} \in \mathrm{C}_{\mathrm{K}_{-}} ; \\
0, & t \in \mathrm{C}_{\mathrm{K}_{-}}, t^{\prime} \in \mathrm{C}_{\mathrm{K}_{+}} ; \\
0, & t \in \mathrm{C}_{\mathrm{K}_{+}}, t^{\prime} \in \mathrm{C}_{\mathrm{K}_{-}} ; \\
-\delta\left(t-t^{\prime}\right), & t \in \mathrm{C}_{\mathrm{K}_{+}}, t^{\prime} \in \mathrm{C}_{\mathrm{K}_{+}} .\end{cases}
\end{aligned}
$$

In general a function $\mathrm{F}(t, t)$ defined on the Keldysh contour is given by

$$
\mathrm{F}\left(t, t^{\prime}\right) \equiv \Theta\left(t, t^{\prime}\right) \mathrm{F}^{>}\left(t, t^{\prime}\right)+\Theta\left(t^{\prime}, t\right) \mathrm{F}^{<}\left(t, t^{\prime}\right)
$$

where $\mathrm{F}^{>}\left(t, t^{\prime}\right)$ is the so-called greater component (greater Keldysh-Green function) and $\mathrm{F}^{<}\left(t, t^{\prime}\right)$ is the lesser component (lesser Keldysh-Green function) of $\mathrm{F}\left(t, t^{\prime}\right)$.

Directly calculations can be carried out, in this way by deriving $t$ or $t^{\prime}$ and using Heisenberg equation of motion for the time evolution of the operators, $A(t)=$ $\exp (\mathrm{i} H t) A \exp (-\mathrm{i} H t)$, as

$$
\frac{\partial A(t)}{\partial t}=-\mathrm{i}[A(t), H]
$$
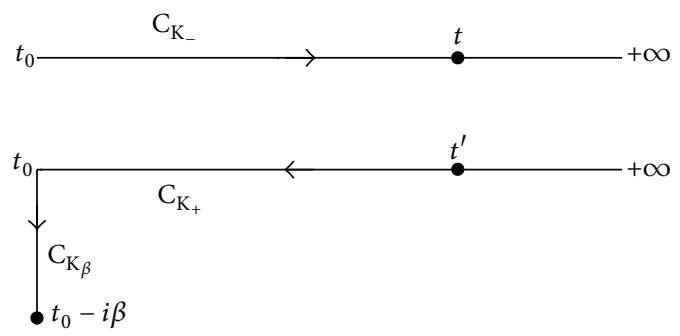

FIGURE 15: The contour $\mathrm{C}_{\mathrm{K}}=\mathrm{C}_{\mathrm{K}_{-}} \cup \mathrm{C}_{\mathrm{K}_{+}}$runs on the real axis, but for clarity its two branches $\mathrm{C}_{\mathrm{K}_{-}}$and $\mathrm{C}_{\mathrm{K}_{+}}$are shown slightly away from the real axis. The contour $\mathrm{C}_{\mathrm{K}}$ runs from $t_{0}$ and returns to $t_{0}$.

and one obtains

$$
\begin{aligned}
& \mathrm{i} \frac{\partial \mathrm{G}\left(t, t^{\prime}\right)}{\partial t} \\
& \quad=\delta\left(t, t^{\prime}\right)\left\langle[A(t), B(t)]_{\mp}\right\rangle-\mathrm{i}\left\langle\mathrm{T}_{\mathrm{K}}[A(t), H] B\left(t^{\prime}\right)\right\rangle, \\
& -\mathrm{i} \frac{\partial \mathrm{G}\left(t, t^{\prime}\right)}{\partial t} \\
& \quad=\delta\left(t, t^{\prime}\right)\left\langle[A(t), B(t)]_{\mp}\right\rangle+\mathrm{i}\left\langle\mathrm{T}_{\mathrm{K}} A(t)\left[B\left(t^{\prime}\right), H\right]\right\rangle .
\end{aligned}
$$

If there is not an applied potential, that is, if $V_{s}=V_{d}=0$, (see Figure 2), the whole system is at equilibrium, and one can use the usual commutator Green functions or equivalently the Matsubara or Temperature Green function to quantify correlations functions. In that case, (A.1) depends only on the time difference $\left(t-t^{\prime}\right)$. However, for times $t>t_{0}$, once the potential difference has been applied, the simple dependence on the time differences no longer holds which signalizes a nonequilibrium situation. In that case, the Keldysh method applies.

\section{B. Equivalent Integral Equation on the Keldysh Countour on Figure 15}

We encounter two cases (see (26) and (42)) where the general strategy to find an integral expression for the KeldyshGreen functions is the following: (1) one first considers the equation of motion for for the Keldysh-Green function of each system (two leads and the QD) (see (25) and (38)). (2) The resulting equation of motion in each case has a delta function as inhomogeneity. (3) The systems are connected in at $t_{0}$, we have as a result two coupled equations of motion (see (20) and (41)). (4) These equations are converted into an equivalent integral equation on the Keldysh contour. (5) Straightforwardly, derivation of the integral equations results in the differential equation of motion.

\section{Calculation of Convolutions}

In this appendix, we explain how we evaluate the some important convolutions used to calculate lesser KeldyshGreen functions [48, 49]. In general, a function given as 
a convolution on the Keldysh contour poses the definition given in Appendix A. One encounters situations where a Keldysh-Green function is given by a convolution of two other functions:

$$
\mathrm{P}\left(t, t^{\prime}\right)=\int_{\mathrm{C}_{\mathrm{K}}} \mathrm{d}^{\prime \prime} \mathrm{F}\left(t, t^{\prime \prime}\right) \mathrm{G}\left(t^{\prime \prime}, t^{\prime}\right) .
$$

However, for evaluation of quantities like, for example, the current, one needs

$$
\mathrm{P}^{<}\left(t, t^{\prime}\right)=\left.\int_{\mathrm{C}_{\mathrm{K}}} \mathrm{d}^{\prime \prime} \mathrm{F}\left(t, t^{\prime \prime}\right) \mathrm{G}\left(t^{\prime \prime}, t^{\prime}\right)\right|_{t<\mathrm{C}_{\mathrm{K}}} t^{\prime}
$$

One sees that the relative position of $t$ and $t^{\prime}$ divides the contour in three regions of integration: (1) $t^{\prime \prime}<_{\mathrm{C}_{\mathrm{K}}} t$, (2) $t<_{\mathrm{C}_{\mathrm{K}}} t^{\prime \prime}<$ $t^{\prime}$, and (3) $t^{\prime \prime}>_{\mathrm{C}_{\mathrm{K}}} t^{\prime}$. This traduces into the following integrals:

$$
\begin{aligned}
\mathrm{P}^{<}\left(t, t^{\prime}\right)= & \left.\int_{t_{0}}^{t} \mathrm{~d} t^{\prime \prime} \mathrm{F}^{>}\left(t, t^{\prime \prime}\right) \mathrm{G}^{<}\left(t^{\prime \prime}, t^{\prime}\right)\right|_{t^{\prime \prime}<\mathrm{C}_{\mathrm{K}} t} \\
& +\left.\int_{t}^{t^{\prime}} \mathrm{d} t^{\prime \prime} \mathrm{F}^{<}\left(t, t^{\prime \prime}\right) \mathrm{G}^{<}\left(t^{\prime \prime}, t^{\prime}\right)\right|_{t<\mathrm{C}_{\mathrm{K}}} t^{\prime \prime}<t^{\prime} \\
& +\left.\int_{t^{\prime}}^{t_{0}} \mathrm{~d} t^{\prime \prime} \mathrm{F}^{<}\left(t, t^{\prime \prime}\right) \mathrm{G}^{>}\left(t^{\prime \prime}, t^{\prime}\right)\right|_{t^{\prime \prime}>\mathrm{C}_{\mathrm{K}} t^{\prime}} .
\end{aligned}
$$

With bookkeeping manipulations of the second integral, one obtains

$$
\begin{aligned}
\mathrm{P}^{<} & \left(t, t^{\prime}\right) \\
& =\int_{t_{0}}^{t_{1}} \mathrm{~d} t^{\prime \prime}\left[\mathrm{F}^{(\mathrm{r})}\left(t, t^{\prime \prime}\right) \mathrm{G}^{<}\left(t^{\prime \prime}, t^{\prime}\right)+\mathrm{F}^{<}\left(t, t^{\prime \prime}\right) \mathrm{G}^{(\mathrm{a})}\left(t^{\prime \prime}, t^{\prime}\right)\right],
\end{aligned}
$$

where the retarded Keldysh-Green function $\mathrm{F}^{(\mathrm{r})}\left(t, t^{\prime \prime}\right)$ and the advance Keldysh-Green function $\mathrm{G}^{(\mathrm{a})}\left(t, t^{\prime \prime}\right)$ are given by

$$
\begin{gathered}
\mathrm{F}^{(\mathrm{r})}\left(t, t^{\prime \prime}\right)=\Theta\left(t-t^{\prime \prime}\right)\left[\mathrm{F}^{>}\left(t, t^{\prime \prime}\right)-\mathrm{F}^{<}\left(t, t^{\prime \prime}\right)\right], \\
\mathrm{G}^{(\mathrm{a})}\left(t^{\prime \prime}, t^{\prime}\right)=-\Theta\left(t^{\prime}-t^{\prime \prime}\right)\left[\mathrm{G}^{>}\left(t^{\prime \prime}, t^{\prime}\right)-\mathrm{G}^{<}\left(t^{\prime \prime}, t^{\prime}\right)\right] .
\end{gathered}
$$

In the said way of reasoning one obtains

$$
\begin{aligned}
\mathrm{P}^{<\{>\}}\left(t, t^{\prime}\right)=\int_{-\infty}^{\infty} \mathrm{d} t^{\prime \prime}[ & \mathrm{F}^{(\mathrm{r})}\left(t, t^{\prime \prime}\right) \mathrm{G}^{<\{>\}}\left(t^{\prime \prime}, t^{\prime}\right) \\
+ & \left.\mathrm{F}^{<\{>\}}\left(t, t^{\prime \prime}\right) \mathrm{G}^{(\mathrm{a})}\left(t^{\prime \prime}, t^{\prime}\right)\right] .
\end{aligned}
$$

With the definitions of a retarded/advanced Keldysh-Green function one easily obtains for $\mathrm{P}^{(\mathrm{r})}\left(t, t^{\prime}\right)$

$$
\begin{aligned}
& \mathrm{P}^{(\mathrm{r})}\left(t, t^{\prime}\right) \\
& =\Theta\left(t-t^{\prime}\right) \int_{-\infty}^{\infty} \mathrm{d} t^{\prime \prime} \mathrm{F}^{(\mathrm{r})}\left(t, t^{\prime \prime}\right)\left[\mathrm{G}^{>}\left(t^{\prime \prime}-t^{\prime}\right)-\mathrm{G}^{<}\left(t^{\prime \prime}, t^{\prime}\right)\right] \\
& \quad+\Theta\left(t^{\prime}-t\right) \int_{-\infty}^{\infty} \mathrm{d} t^{\prime \prime}\left[\mathrm{F}^{>}\left(t-t^{\prime \prime}\right)-\mathrm{F}^{<}\left(t, t^{\prime \prime}\right)\right] \mathrm{G}^{(\mathrm{r})}\left(t^{\prime \prime}, t^{\prime}\right),
\end{aligned}
$$

and therefore

$$
\mathrm{P}^{(\mathrm{r})}\left(t, t^{\prime}\right)=\int_{-\infty}^{\infty} \mathrm{d} t^{\prime \prime} \mathrm{F}^{(\mathrm{r})}\left(t, t^{\prime \prime}\right) \mathrm{G}^{(\mathrm{r})}\left(t^{\prime \prime}, t^{\prime}\right) .
$$

Similarly for $\mathrm{P}^{(\mathrm{a})}\left(t, t^{\prime}\right)$ one obtains

$$
\mathrm{P}^{(\mathrm{a})}\left(t, t^{\prime}\right)=\int_{-\infty}^{\infty} \mathrm{d} t^{\prime \prime} \mathrm{F}^{(\mathrm{a})}\left(t, t^{\prime \prime}\right) \mathrm{G}^{(\mathrm{a})}\left(t^{\prime \prime}, t^{\prime}\right) \text {. }
$$

\section{Calculation of Unperturbed Green Functions and Retarded Self-Energy}

Below, we proceed to evaluate the unperturbed Green functions $\mathrm{g}_{\eta \vec{k} \sigma}$ and $\mathrm{f}_{\eta \vec{k} \sigma}$. To achieve this, it is necessary to introduce the chemical potential shift in each superconductor,

$$
\mathscr{H}_{\eta}=H_{\eta}-\mu_{\eta} N_{\eta}
$$

so that

$$
\begin{aligned}
a_{\eta \vec{k} \sigma}(t) & \equiv \mathrm{e}^{\mathrm{i} H_{\eta} t} a_{\eta \vec{k} \sigma} \mathrm{e}^{-\mathrm{i} H_{\eta} t} \\
& =\mathrm{e}^{\mathrm{i} \mathscr{H}_{\eta} t}\left(\mathrm{e}^{\mathrm{i} \mu_{\eta} t} a_{\eta \vec{k} \sigma} \mathrm{e}^{-\mathrm{i} \mu_{\eta} t}\right) \mathrm{e}^{-\mathrm{i} \mathscr{H} \eta_{\eta} t} \\
& =\mathrm{e}^{-\mathrm{i} \mu_{\eta} t}\left(\mathrm{e}^{\mathrm{i} \mathscr{H}{ }_{\eta} t} a_{\eta \vec{k} \sigma} \mathrm{e}^{-\mathrm{i} \mathscr{H} t}\right) \longrightarrow \mathrm{e}^{-\mathrm{i} \mu_{\eta} t} a_{\eta \vec{k} \sigma}(t),
\end{aligned}
$$

due to

$$
\begin{aligned}
& \mathrm{e}^{\mathrm{i} \mathscr{H}_{\eta} t} a_{\eta \vec{k} \sigma} \mathrm{e}^{-\mathrm{i} \mathscr{H}_{\eta} t}=a_{\eta \vec{k} \sigma} \mathrm{e}^{-\mathrm{i} \mathrm{E}_{\eta \vec{k}} t}, \\
& \mathrm{e}^{\mathrm{i} N_{\eta} t} a_{\eta \vec{k} \sigma} \mathrm{e}^{-\mathrm{i} N_{\eta} t}=a_{\eta \vec{k} \sigma} \mathrm{e}^{-\mathrm{i} \mu_{\eta \vec{k}} t},
\end{aligned}
$$

because $\left[N_{\eta}, H_{\eta}\right]=0$. Consequently, the first unperturbed retarded Green function $\mathrm{g}_{\eta \vec{k} \sigma}^{(\mathrm{r})}\left(t, t^{\prime}\right)$ is given by

$$
\begin{aligned}
\mathrm{g}_{\eta \vec{k} \sigma}^{(\mathrm{r})}\left(t, t^{\prime}\right) & \\
= & -\mathrm{i} \Theta\left(t-t^{\prime}\right)\left\langle\left\{\mathrm{e}^{-\mathrm{i} \mu_{\eta} t} a_{\eta \vec{k} \sigma}(t), \mathrm{e}^{\mathrm{i} \mu_{\eta} t^{\prime}} a_{\eta \vec{k} \sigma}^{\dagger}\left(t^{\prime}\right)\right\}\right\rangle \\
= & -\mathrm{i} \Theta\left(t-t^{\prime}\right) \mathrm{e}^{-\mathrm{i} \mu_{\eta}\left(t-t^{\prime}\right)} \\
& \times\left\langle\left\{ u_{\eta \vec{k}} \gamma_{\eta \vec{k} \sigma}(t)+\sigma v_{\eta \vec{k}} \gamma_{\eta \vec{k} \sigma}^{\dagger}(t), u_{\eta \vec{k}} \gamma_{\eta \vec{k} \sigma}^{\dagger}\left(t^{\prime}\right)\right.\right. \\
& \left.\left.+\sigma v_{\eta \vec{k}} \gamma_{\eta-\vec{k}-\sigma}\left(t^{\prime}\right)\right\}\right\rangle \\
= & -\mathrm{i} \Theta\left(t-t^{\prime}\right) \mathrm{e}^{-\mathrm{i} \mu_{\eta}\left(t-t^{\prime}\right)}\left[u_{\eta \vec{k}}^{2} \mathrm{e}^{-\mathrm{i} \mathrm{E}_{\eta \vec{k}}\left(t-t^{\prime}\right)}+v_{\eta \vec{k}}^{2} \mathrm{e}^{\mathrm{i} \mathrm{E}_{\eta \vec{k}}\left(t-t^{\prime}\right)}\right], \\
= & -\mathrm{i} \Theta\left(t-t^{\prime}\right)\left[u_{\eta \vec{k}}^{2} \mathrm{e}^{-\mathrm{i}\left(E_{\eta \vec{k}}+\mu_{\eta}\right)\left(t-t^{\prime}\right)}+v_{\eta \vec{k}}^{2} \mathrm{e}^{\mathrm{i}\left(\mathrm{E}_{\eta \vec{k}}-\mu_{\eta}\right)\left(t-t^{\prime}\right)}\right],
\end{aligned}
$$

where the fermion operators $\gamma_{\eta \vec{k} \sigma}^{\dagger}, \gamma_{\eta \vec{k} \sigma}$ create and annihilate the "Bogoliubov quasi-particles" and

$$
\sigma= \begin{cases}\uparrow & =1 \\ \downarrow & =-1\end{cases}
$$


the spin index. They will be linear combinations of the creation and annihilation operators of the real electrons:

$$
\begin{gathered}
a_{\eta \vec{k} \sigma}(t)=u_{\eta \vec{k}} \gamma_{\eta \vec{k} \sigma}(t)+\sigma v_{\eta \vec{k}} \gamma_{\eta \vec{k} \sigma}^{\dagger}(t), \\
a_{\eta \vec{k} \sigma}^{\dagger}\left(t^{\prime}\right)=u_{\eta \vec{k}} \gamma_{\eta \vec{k} \sigma}^{\dagger}\left(t^{\prime}\right)+\sigma v_{\eta \vec{k}} \gamma_{\eta-\vec{k}-\sigma}\left(t^{\prime}\right) .
\end{gathered}
$$

Applying the Fourier transformations to $\mathrm{g}_{\eta \vec{k} \sigma}^{(\mathrm{r})}\left(t, t^{\prime}\right)$

$$
\mathrm{g}_{\eta \vec{k}}^{(\mathrm{r})}\left(t, t^{\prime}\right)=\int_{-\infty}^{\infty} \frac{\mathrm{d} \omega}{2 \pi} \mathrm{e}^{-\mathrm{i} \omega\left(t-t^{\prime}\right)} \mathrm{g}_{\eta \vec{k}}^{(\mathrm{r})}(\omega),
$$

with

$$
\mathrm{g}_{\eta \vec{k}}^{(\mathrm{r})}(\omega)=\frac{u_{\eta \vec{k}}^{2}}{\omega-\mathrm{E}_{\eta \vec{k}}-\mu_{\eta}+\mathrm{i} 0^{+}}+\frac{v_{\eta \vec{k}}^{2}}{\omega+\mathrm{E}_{\eta \vec{k}}-\mu_{\eta}+\mathrm{i} 0^{+}} .
$$

Therefore we have

$$
\begin{aligned}
& V_{\eta}^{2} \sum_{\vec{k}} \mathrm{~g}_{\eta \vec{k}}^{(\mathrm{r})}(\omega) \\
&=\frac{1}{2} V_{\eta}^{2} \sum_{\vec{k}}\left(\frac{1}{\omega-\mathrm{E}_{\eta \vec{k}}-\mu_{\eta}+\mathrm{i} 0^{+}}+\frac{1}{\omega+\mathrm{E}_{\eta \vec{k}}-\mu_{\eta}+\mathrm{i} 0^{+}}\right) \\
&= V_{\eta}^{2} \mathrm{P} \sum_{\vec{k}} \frac{\omega-\mu_{\eta}}{\left(\omega-\mu_{\eta}\right)^{2}-\mathrm{E}_{\eta \vec{k}}^{2}} \\
&-\frac{1}{2} \mathrm{i} \pi V_{\eta}^{2} \sum_{\vec{k}}\left[\delta\left(\omega-\mu_{\eta}-\mathrm{E}_{\eta \vec{k}}\right)+\delta\left(\omega-\mu_{\eta}+\mathrm{E}_{\eta \vec{k}}\right)\right] \\
&=-N_{\eta}(0) V_{\eta}^{2} \mathrm{P} \int_{-\infty}^{\infty} \mathrm{d} \xi \frac{\omega-\mu_{\eta}}{\xi^{2}+\Delta^{2}-(\omega-\mu)^{2}} \\
&-\frac{1}{2} \mathrm{i} \pi V_{\eta}^{2} \sum_{\vec{k}} \delta\left(\left|\omega-\mu_{\eta}\right|-\mathrm{E}_{\eta \vec{k}}\right) .
\end{aligned}
$$

Finally,

$$
\begin{array}{r}
\Sigma^{(\mathrm{r})}(\omega) \equiv V_{\eta}^{2} \sum_{\vec{k}} \mathrm{~g}_{\eta \vec{k}}^{(\mathrm{r})}(\omega)=-\Gamma_{\eta}\left[\frac{\omega-\mu_{\eta}}{\Delta_{\eta}} \zeta\left(\Delta_{\eta}, \omega-\mu_{\eta}\right)\right. \\
\left.+\mathrm{i} \zeta\left(\omega-\mu_{\eta}, \Delta_{\eta}\right)\right]
\end{array}
$$

where

$$
\begin{aligned}
& \Gamma_{\eta}=\pi N_{\eta}\left(\omega-\mu_{\eta}\right) V_{\eta}^{2} \approx \pi N_{\eta}(0) V_{\eta}^{2}, \\
& \zeta\left(\omega, \omega^{\prime}\right) \equiv \Theta\left(|\omega|-\left|\omega^{\prime}\right|\right) \frac{|\omega|}{\sqrt{\omega^{2}-\omega^{\prime 2}}} .
\end{aligned}
$$

$\Gamma_{\eta}=\pi N_{\eta}\left(\omega-\mu_{\eta}\right) V_{\eta}^{2} \approx \pi N_{\eta}(0) V_{\eta}^{2}$ are the coupling constants between the leads and the quantum dot in the wide band limit (WBL). $N_{\eta}(0)$ is the density of states at the $\eta$ Fermi level and $\mathrm{f}(\omega)$ is the Fermi-Dirac distribution function.
The WBL means that the width of the electronic energy bands of the leads is the largest energy. The density of states in the contacts varies on a scale of Fermi energy. These scales are of order $1-10 \mathrm{eV}\left(\sim 10^{4}-10^{5} \mathrm{~K}\right)$ which are much larger than the energies involved in the quantum dot $\sim \mathrm{meV} \sim 10 \mathrm{~K}$. Furthermore, $\Gamma_{\eta}\left(\omega-\mu_{\eta}\right)$ varies slowly with $\omega-\mu_{\eta}$ and the prefactor $\mathscr{D}_{\eta}\left(\omega-\mu_{\eta}\right)$ varies in the range of wide band and changes $\omega-\mu_{\eta}$ on the average of $\left|V_{\eta \vec{k}}\right|^{2}$ occur on the order of meV. Therefore, we ignore the dependence of $\omega-\mu_{\eta}$ in $\Gamma_{\eta}\left(\omega-\mu_{\eta}\right)$. The WBL establishes that an electron in the dot decays in an continuum of states of the leads and is sufficient condition for the existence of a stationary state, as has been shown rigorously in [50].

\section{E. Calculation of the Lesser Green Function and Lesser Self-Energy}

Consider

$$
\begin{gathered}
\mathrm{g}_{\eta \vec{k} \sigma}^{<}\left(t, t^{\prime}\right) \\
=\mathrm{i}\left\langle\left\{\mathrm{e}^{\mathrm{i} \mu_{\eta} t^{\prime}} a_{\eta \vec{k} \sigma}^{\dagger}\left(t^{\prime}\right), \mathrm{e}^{-\mathrm{i} \mu_{\eta} t} a_{\eta \vec{k} \sigma}(t)\right\}\right\rangle \\
=\mathrm{ie} \mu^{\mathrm{i} \mu_{\eta}\left(t-t^{\prime}\right)}\left\langle\left[u_{\eta \vec{k}} \gamma_{\eta \vec{k} \sigma}^{\dagger}\left(t^{\prime}\right)+\sigma v_{\eta \vec{k}} \gamma_{\eta-\vec{k}-\sigma}\left(t^{\prime}\right)\right]\right. \\
\left.\times\left[u_{\eta \vec{k}} \gamma_{\eta \vec{k} \sigma}(t)+\sigma v_{\eta \vec{k}} \gamma_{\eta-\vec{k}-\sigma}^{\dagger}(t)\right]\right\rangle \\
=\mathrm{i}\left[u_{\eta \vec{k}}^{2} \mathrm{e}^{-\mathrm{i}\left(\mathrm{E}_{\eta \vec{k}}+\mu_{\eta}\right)\left(t-t^{\prime}\right)} \mathrm{f}\left(\mathrm{E}_{\eta \vec{k}}\right)\right. \\
\left.+v_{\eta \vec{k}}^{2} \mathrm{e}^{\mathrm{i}\left(\mathrm{E}_{\eta \vec{k}}-\mu_{\eta}\right)\left(t-t^{\prime}\right)} \mathrm{f}\left(-\mathrm{E}_{\eta \vec{k}}\right)\right] .
\end{gathered}
$$

Applying the Fourier transformations,

$$
\mathrm{g}_{\eta \vec{k} \sigma}^{<}\left(t, t^{\prime}\right)=\int_{-\infty}^{\infty} \frac{\mathrm{d} \omega}{2 \pi} \mathrm{e}^{-\mathrm{i} \omega\left(t-t^{\prime}\right)} \mathrm{g}_{\eta \vec{k}}^{<}(\omega),
$$

with

$$
\begin{aligned}
\mathrm{g}_{\eta \vec{k} \sigma}^{<}(\omega)=2 \pi \mathrm{i}[ & u_{\eta \vec{k}}^{2} \delta\left(\omega-\mathrm{E}_{\eta \vec{k}}-\mu_{\eta}\right) \\
& \left.+v_{\eta \vec{k}}^{2} \delta\left(\omega+\mathrm{E}_{\eta \vec{k}}-\mu_{\eta}\right)\right] \mathrm{f}\left(\omega-\mu_{\eta}\right) .
\end{aligned}
$$

Therefore we have

$$
\begin{gathered}
V_{\eta}^{2} \sum_{\vec{k}} \mathrm{~g}_{\eta \vec{k} \sigma}^{<}(\omega) \\
=2 \pi \mathrm{i} V_{\eta}^{2} \sum_{\vec{k}}\left[u_{\eta \vec{k}}^{2} \delta\left(\omega-\mathrm{E}_{\eta \vec{k}}-\mu_{\eta}\right)\right. \\
\left.+v_{\eta \vec{k}}^{2} \delta\left(\omega+\mathrm{E}_{\eta \vec{k}}-\mu_{\eta}\right)\right] \times \mathrm{f}\left(\omega-\mu_{\eta}\right) \\
=2 \pi \mathrm{i} V_{\eta}^{2} \frac{1}{2} \sum_{\vec{k}} \delta\left(\left|\omega-\mu_{\eta}\right|-\mathrm{E}_{\eta \vec{k}}\right) \mathrm{f}\left(\omega-\mu_{\eta}\right) .
\end{gathered}
$$


Finally,

$\Sigma^{<}(\omega) \equiv V_{\eta}^{2} \sum_{\vec{k}} \mathrm{~g}_{\eta \vec{k}}^{<}(\omega)=2 \mathrm{i} \Gamma_{\eta} \zeta\left(\omega-\mu_{\eta}, \Delta_{\eta}\right) \mathrm{f}\left(\omega-\mu_{\eta}\right)$.

\section{F. Calculation of the Retarded}

(Tilde) Self-Energy

Consider

$$
\begin{aligned}
\left.\widetilde{\mathrm{f}}_{\eta \vec{k} \sigma}^{\mathrm{r}}\right)( & \left.t, t^{\prime}\right) \\
= & -\mathrm{i} \Theta\left(t-t^{\prime}\right)\left\langle\left\{\mathrm{e}^{\mathrm{i} \mu_{\eta} t} a_{\eta \vec{k} \sigma}(t), \mathrm{e}^{\mathrm{i} \mu_{\eta} t^{\prime}} a_{\eta-\vec{k}-\sigma}^{\dagger}\left(t^{\prime}\right)\right\}\right\rangle \\
= & -\mathrm{i} \Theta\left(t+t^{\prime}\right) \mathrm{e}^{\mathrm{i} \mu_{\eta}\left(t+t^{\prime}\right)} \\
& \times\left\langle\left\{ u_{\eta \vec{k}} \gamma_{\eta \vec{k} \sigma}^{\dagger}(t)+\sigma v_{\eta \vec{k}} \gamma_{\eta \vec{k} \sigma}^{\dagger}(t), u_{\eta \vec{k}} \gamma_{\eta-\vec{k}-\sigma}\left(t^{\prime}\right)\right.\right. \\
& \left.\left.+\sigma v_{\eta \vec{k}} \gamma_{\eta \vec{k} \sigma}^{\dagger}\left(t^{\prime}\right)\right\}\right\rangle \\
= & -\mathrm{i} \Theta\left(t-t^{\prime}\right) \mathrm{e}^{-2 \mathrm{i} \mu_{\eta} t} \mathrm{e}^{-\mathrm{i} \mu_{\eta}\left(t-t^{\prime}\right)} \sigma v_{\eta \vec{k}} u_{\eta \vec{k}} \\
& \times\left[\mathrm{e}^{\mathrm{i} E_{\vec{k}}\left(t-t^{\prime}\right)}+\mathrm{e}^{\mathrm{i} \mathrm{E}_{\eta \vec{k}}\left(t-t^{\prime}\right)}\right] \\
= & -\mathrm{i} \Theta\left(t-t^{\prime}\right) \frac{\sigma \Delta_{\eta}}{2 \mathrm{E}_{\eta \vec{k}}} \\
& \times\left[\mathrm{e}^{-\mathrm{i}\left(\mathrm{E}_{\eta \vec{k}}-\mu_{\eta}\right)\left(t-t^{\prime}\right)}+\mathrm{e}^{\mathrm{i}\left(\mathrm{E}_{\eta \vec{k}}+\mu_{\eta}\right)\left(t-t^{\prime}\right)}\right] .
\end{aligned}
$$

Applying the Fourier transformations

$$
V_{\eta}^{2} \sum_{\vec{k}}^{-(\mathrm{r})}\left(t \vec{k} \sigma=t^{\prime}\right)=\int_{-\infty}^{\infty} \mathrm{e}^{-2 \mathrm{i} \mu_{\eta} t} \sigma \frac{\mathrm{d} \omega}{2 \pi} \mathrm{e}^{-\mathrm{i} \omega\left(t-t^{\prime}\right)} \widetilde{\Xi}_{\eta \vec{k}}^{(\mathrm{r})}(\omega),
$$

with

$$
\begin{aligned}
& V_{\eta \vec{k}}^{2} \sum_{\vec{k}} \widetilde{\Xi}_{\eta \vec{k}}^{(\mathrm{r})}(\omega) \\
& =\frac{\Delta_{\eta}}{2 \mathrm{E}_{\eta \vec{k}}}\left[\frac{1}{\omega-\mathrm{E}_{\eta \vec{k}}-\mu_{\eta}+\mathrm{i} 0^{+}}+\frac{1}{\omega+\mathrm{E}_{\eta \vec{k}}-\mu_{\eta}+\mathrm{i} 0^{+}}\right] \\
& =V_{\eta}^{2} \sum_{\vec{k}} \frac{\Delta_{\eta}}{2 \mathrm{E}_{\eta \vec{k}}}\left[\frac{1}{\omega-\mathrm{E}_{\eta \vec{k}}-\mu_{\eta}+\mathrm{i} 0^{+}}+\frac{1}{\omega+\mathrm{E}_{\eta \vec{k}}-\mu_{\eta}+\mathrm{i} 0^{+}}\right] \\
& =V_{\eta}^{2} \Delta_{\eta} \sum_{\vec{k}}\left[\mathrm{P} \frac{1}{\xi_{\vec{k}}^{2}+\Delta_{\eta}^{2}-\left(\omega-\mu_{\eta}\right)^{2}}+\frac{\mathrm{i} \pi}{2\left(\omega-\mu_{\eta}\right)}\right. \\
& \left.\quad \times \delta\left(\left|\omega+\mu_{\eta}\right|-\mathrm{E}_{\eta \vec{k}}\right)\right]
\end{aligned}
$$

$$
\begin{aligned}
= & N_{\eta}(0) V_{\eta}^{2} \Delta_{\eta} \times \mathrm{P} \int_{-\infty}^{\infty} \mathrm{d} \xi \frac{1}{\xi^{2}+\Delta^{2}-(\omega+\mu)^{2}} \\
& +\frac{\mathrm{i} \pi V_{\eta}^{2}}{2\left(\omega+\mu_{\eta}\right)} \sum_{\vec{k}} \delta\left(\left|\omega+\mu_{\eta}\right|-\mathrm{E}_{\eta \vec{k}}\right) .
\end{aligned}
$$

Finally,

$$
\widetilde{\Xi}_{\eta \vec{k}}^{(\mathrm{r})}(\omega)=\Gamma_{\eta}\left[\zeta\left(\Delta_{\eta}, \omega+\mu_{\eta}\right)+\mathrm{i} \frac{\Delta_{\eta}}{\omega+\mu_{\eta}} \zeta\left(\omega+\mu_{\eta}, \Delta_{\eta}\right)\right] .
$$

\section{G. Calculation of the Lesser (Tilde) Self-Energy} Consider

$$
\begin{gathered}
\widetilde{\mathrm{f}}_{\eta \vec{k} \sigma}\left(t, t^{\prime}\right) \\
=\mathrm{i}\left\langle\left\{\mathrm{e}^{-\mathrm{i} \mu_{\eta} t^{\prime}} a_{\eta-\vec{k}-\sigma}\left(t^{\prime}\right), \mathrm{e}^{-\mathrm{i} \mu_{\eta} t} a_{\eta \vec{k} \sigma}(t)\right\}\right\rangle \\
=\mathrm{i} \mathrm{e}^{-\mathrm{i} \mu_{\eta}\left(t+t^{\prime}\right)}\left\langle\left[u_{\eta \vec{k}} \gamma_{\eta-\vec{k}-\sigma}\left(t^{\prime}\right)-\sigma v_{\eta \vec{k}} \gamma_{\eta-\vec{k}-\sigma}^{\dagger}\left(t^{\prime}\right)\right]\right. \\
\left.\times\left[u_{\eta \vec{k}} \gamma_{\eta \vec{k} \sigma}(t)+\sigma v_{\eta \vec{k}} \gamma_{\eta-\vec{k}-\sigma}^{\dagger}(t)\right]\right\rangle \\
=\mathrm{ie}^{-2 \mathrm{i} \mu_{\eta} t} \mathrm{e}^{\mathrm{i} \mu_{\eta}\left(t-t^{\prime}\right)}\left[\sigma u_{\eta \vec{k}} v_{\eta \vec{k}} \mathrm{e}^{\mathrm{i}\left(\mathrm{E}_{\eta \vec{k}}\right)\left(t-t^{\prime}\right)} \mathrm{f}\left(-\mathrm{E}_{\eta \vec{k}}\right)\right. \\
\left.+\sigma v_{\eta \vec{k}} u_{\eta \vec{k}} \mathrm{e}^{-\mathrm{i}\left(\mathrm{E}_{\eta \vec{k}}\right)\left(t-t^{\prime}\right)} \mathrm{f}\left(\mathrm{E}_{\eta \vec{k}}\right)\right] \\
=\mathrm{ie}^{-2 \mathrm{i} \mu_{\eta} t} \sigma \frac{\Delta_{\eta}}{2 \mathrm{E}_{\vec{\eta}}}\left[\mathrm{e}^{\mathrm{i}\left(\mathrm{E}_{\eta \vec{k}}+\mu_{\eta}\right)\left(t-t^{\prime}\right)} \mathrm{f}\left(-\mathrm{E}_{\eta \vec{k}}\right)\right. \\
\left.+\mathrm{e}^{-\mathrm{i}\left(\mathrm{E}_{\eta \vec{k}}-\mu_{\eta}\right)\left(t-t^{\prime}\right)} \mathrm{f}\left(\mathrm{E}_{\eta \vec{k}}\right)\right] .
\end{gathered}
$$

Therefore we have

$$
V_{\eta}^{2} \sum_{\vec{k}}^{\widetilde{\mathrm{f}_{\eta \vec{k}}}}\left(t, t^{\prime}\right)=\int_{-\infty}^{\infty} \mathrm{e}^{-2 \mathrm{i} \mu_{\eta} t} \sigma \frac{\mathrm{d} \omega}{2 \pi} \mathrm{e}^{-\mathrm{i} \omega\left(t-t^{\prime}\right)} \widetilde{\Xi}_{\eta \vec{k}}^{<}(\omega),
$$

with

$$
\begin{aligned}
& \widetilde{\Xi}_{\eta \vec{k}}^{<}(\omega) \\
& =V_{\eta}^{2} \sum_{\vec{k}} \frac{\pi \mathrm{i}}{\mathrm{E}_{\eta \vec{k}}}\left[\delta\left(\omega+\mathrm{E}_{\eta \vec{k}}+\mu_{\eta}\right) \mathrm{f}\left(-\mathrm{E}_{\eta \vec{k}}\right)\right. \\
& \left.\quad-\delta\left(\omega-\mathrm{E}_{\eta \vec{k}}+\mu_{\eta}\right) \mathrm{f}\left(\mathrm{E}_{\eta \vec{k}}\right)\right] \\
& =V_{\eta}^{2} \sum_{\vec{k}} \frac{\pi \mathrm{i}}{\mathrm{E}_{\eta \vec{k}}}\left[\delta\left(\omega+\mathrm{E}_{\eta \vec{k}}+\mu_{\eta}\right)\right. \\
& \left.\quad-\delta\left(\omega-\mathrm{E}_{\eta \vec{k}}+\mu_{\eta}\right)\right] \mathrm{f}\left(\omega+\mu_{\eta}\right) \\
& =\frac{-2 \mathrm{i} \Delta_{\eta}}{\omega-\mu_{\eta}}\left[\frac{\pi V_{\eta}^{2}}{2} \sum_{\eta} \delta\left(\left|\omega+\mu_{\eta}\right|-\mathrm{E}_{\eta \vec{k}}\right)\right] \mathrm{f}\left(\omega+\mu_{\eta}\right) .
\end{aligned}
$$


Finally,

$$
\widetilde{\Xi}_{\eta \vec{k}}^{<}(\omega)=-2 \mathrm{i} \Gamma_{\eta} \frac{\Delta_{\eta}}{\omega+\mu_{\eta}} \zeta\left(\omega+\mu_{\eta}, \Delta_{\eta}\right) \mathrm{f}\left(\omega+\mu_{\eta}\right) .
$$

\section{Conflict of Interests}

The authors declare that there is no conflict of interests regarding the publication of this paper.

\section{References}

[1] L. P. Kouwenhoven, D. G. Austing, and S. Tarucha, "Few-electron quantum dots," Reports on Progress in Physics, vol. 64, no. 6, pp. 701-736, 2001.

[2] S. De Franceschi, L. Kouwenhoven, C. Schönenberger, and W. Wernsdorfer, "Hybrid superconductor-quantum dot devices," Nature Nanotechnology, vol. 5, no. 10, pp. 703-711, 2010.

[3] M. A. Kastner, "Artificial atoms," Physics Today, vol. 46, no. 1, pp. 24-31, 1993.

[4] S. Datta, Quantum Trasport: Atom to Transistor, Cambridge University Press, Cambridge, UK, 2005.

[5] R. Tidecks, Current-Induced Nonequilibrium Phenomena in Quasi-One-Dimensional Superconductors, vol. 121, Springer Tracts in Modern Physics, 1990.

[6] M. Tinkham, Introduction to Superconductivity, chapter 11, Dover, 2004.

[7] N. Kopnin, Theory of Nonequilibrium Superconductivity, Clarendon Press, Oxford, UK, 2001.

[8] M. Bonitz and D. Semkat, Progress in Nonequilibrium Green's Functions II: Proceeding of the Conference in Dresden, Germany 19-23 August 2002, World Scientific, 2003.

[9] R. van Leeuwen, N. E. Dahlen, G. Stefanucci, C.-O. Almbladh, and U. von Barth, "Introduction to the Keldysh formalism," Lecture Notes in Physics, vol. 706, pp. 33-59, 2006.

[10] F. P. Marin, "Keldysh Elemental," http://fisica.ciens.ucv.ve/ felix/Keldysh/.

[11] N. Vercruyssen, T. G. A. Verhagen, M. G. Flokstra, J. P. Pekola, and T. M. Klapwijk, "Evanescent states and nonequilibrium in driven superconducting nanowires," Physical Review B, vol. 85, no. 22, Article ID 224503, 2012.

[12] M. Serbyn and M. Skorsov, "Onset of superconductivity in a voltage-biased normal-superconducting-normal microbridge," Physical Review B, vol. 87, no. 2, Article ID 020501, 2013.

[13] I. Snyman and Y. V. Nazarov, "Bistability in voltage-biased normal-metal/insulator/superconductor/ insulator/normal-metal structures," Physical Review B, vol. 79, no. 1, Article ID 014510, 2009.

[14] Z. Y. Zeng, B. Li, and F. Claro, "Electronic transport in hybrid mesoscopic structures: a nonequilibrium Green function approach," Physical Review B, vol. 68, no. 11, Article ID 115319, 2003.

[15] M. Krawiec and K. I. Wysokiński, "Electron transport through a strongly interacting quantum dot coupled to a normal metal and BCS superconductor," Superconductor Science and Technology, vol. 17, no. 1, pp. 103-112, 2004.

[16] K. Kang, "Transport through an interacting quantum dot coupled to two superconducting leads," Physical Review B, vol. 57, no. 19, pp. 11891-11894, 1998.
[17] A. Levy Yeyati, J. C. Cuevas, A. López-Dávalos, and A. MartínRodero, "Josephson and Andreev transport through quantum dots," Advances in Physics, vol. 60, no. 6, pp. 899-958, 2011.

[18] A. Martín-Rodero and A. Levy Yeyati, "Resonant tunneling through a small quantum dot coupled to superconducting leads," Physical Review B, vol. 55, no. 10, pp. R6137-R6140, 1997.

[19] M. G. Pala, M. Governale, and J. König, "Nonequilibrium Josephson and Andreev current through interacting quantum dots," New Journal of Physics, vol. 9, no. 8, article 278, 2007.

[20] F. Giazotto, T. T. Heikkilä, F. Taddei, R. Fazio, J. P. Pekola, and F. Beltram, "Tailoring Josephson coupling through superconductivity-induced nonequilibrium," Physical Review Letters, vol. 92, no. 13, Article ID 137001, 2004.

[21] S. Datta, "Fock space formulation for nanoscale electronic transport," 2006, http://arxiv.org/abs/cond-mat/0603034.

[22] S. Datta, Electronic Transport in Mesoscopic Systems, Cambridge University Press, Cambridge, UK, 1997.

[23] A. W. Ghost and S. Datta, "Molecular conduction: paradigms and possibilities," Journal of Computational Electronics, vol. 1, no. 4, pp. 515-525, 2002.

[24] G. C. Liang, A. W. Ghosh, M. Paulsson, and S. Datta, "Electrostatic potential profiles of molecular conductors," Physical Review B, vol. 69, no. 11, Article ID 115302, 2004.

[25] B. Muralidharan, A. W. Ghosh, S. K. Pati, and S. Datta, "Theory of high bias coulomb blockade in ultrashort molecules," IEEE Transactions on Nanotechnology, vol. 6, no. 5, pp. 536-544, 2007.

[26] L. V. Keldysh, "Diagram technique for nonequilibrium processes," Soviet Physics JETP, vol. 20, pp. 1018-1026, 1965.

[27] H. Haug and A. P. Jauho, Quantum Kinetics in Transport and Optics of Semiconductors, Springer, Berlin, Germany, 1996.

[28] D. C. Ralph, C. T. Black, and M. Tinkham, "Spectroscopic measurements of discrete electronic states in single metal particles," Physical Review Letters, vol. 74, no. 16, pp. 3241-3244, 1995.

[29] M. Paulsson, F. Zahid, and S. Datta, "Resistance of a molecule," in Nanoparticle Manipulation by Electrostatic forces MP Hughes Chapter in Nanoscience, Engineering and Technology Handbook, W. Goddard, D. Brenner, S. Lyshevski, and G. Iafrate, Eds., CRC Press, Boca Raton, Fla, USA, 2002.

[30] S. Datta, "Electrical resistance: an atomistic view," Nanotechnology, vol. 15, no. 7, pp. S433-S451, 2004.

[31] T. Christen and M. Büttiker, "Gauge-invariant nonlinear electric transport in mesoscopic conductors," Europhysics Letters, vol. 35, no. 7, pp. 523-528, 1996.

[32] B. Wang, J. Wang, and H. Guo, "Nonlinear I-V characteristics of a mesoscopic conductor," Journal of Applied Physics, vol. 86, no. 9, pp. 5094-5102, 1999.

[33] Y. Meir and N. S. Wingreen, "Landauer formula for the current through an interacting electron region," Physical Review Letters, vol. 68, no. 16, pp. 2512-2515, 1992.

[34] A.-P. Jauho, N. S. Wingreen, and Y. Meir, “Time-dependent transport in interacting and noninteracting resonant-tunneling systems," Physical Review B, vol. 50, no. 8, pp. 5528-5544, 1994.

[35] H. P. Beuer and F. Petruccione, The Theory of Open Quantum Systems, Clarendon Press, Oxford, UK, 2006.

[36] D. S. Kosov, T. Prosen, and B. Zunkovic, "A Markovian kinetic equation approach to electron transport through a quantum dot coupled to superconducting leads," Journal Physics: Condensed Matter, vol. 25, no. 7, Article ID 075702, 2013.

[37] C. T. Black, D. C. Ralph, and M. Tinkham, "Spectroscopy of the superconducting gap in individual nanometer-scale aluminum 
particles," Physical Review Letters, vol. 76, no. 4, pp. 688-691, 1996.

[38] S. Pfaller, A. Donarini, and M. Grifoni, "Subgap features due to quasiparticle tunneling in quantum dots coupled to superconducting leads," Physical Review B, vol. 87, no. 15, Article ID 155439, 2013.

[39] M. Esposito and M. Galperin, "Transport in molecular states language: generalized quantum master equation approach," Physical Review B, vol. 79, no. 20, Article ID 205303, 2009.

[40] P. W. Anderson, "Localized magnetic states in metals," Physical Review, vol. 124, no. 1, pp. 41-53, 1961.

[41] J. Bardeen, L. N. Cooper, and J. R. Schrieffer, “Theory of superconductivity," Physical Review, vol. 108, no. 5, pp. 1175-1204, 1957.

[42] A. F. Andreev, "Thermal conductivity of the intermediate state of superconductors," Soviet Physics JETP, vol. 19, pp. 1228-1231, 1964.

[43] H. Takayanagi, T. Akazaki, and J. Nitta, "Observation of maximum supercurrent quantization in a superconducting quantum point contact," Physical Review Letters, vol. 75, no. 19, pp. 35333536, 1995.

[44] R. Golizadeh-Mojarad and S. Datta, "Nonequilibrium Green's function based models for dephasing in quantum transport," Physical Review B, vol. 75, no. 8, Article ID 081301, 2007.

[45] A. A. Yanik, G. Klimeck, and S. Datta, "Quantum transport with spin dephasing: a nonequlibrium Green's function approach," Physical Review B, vol. 76, no. 4, Article ID 045213, 2007.

[46] R. Rangel and E. Medina, "Persistent non-ergodic fluctuations in mesoscopic insulators: the NSS model in the unitary and symplectic ensembles," European Physical Journal B, vol. 30, no. 2, pp. 101-109, 2002.

[47] C. B. Whan and T. P. Orlando, "Transport properties of a quantum dot with superconducting leads," Physical Review B, vol. 54, no. 8, pp. R5255-R5258, 1996.

[48] D. C. Langreth, "Linear and nonlinear response theory with applications," Linear and Nonlinear Electron Transport in Solids Nato Advanced Study Institutes Series, vol. 17, pp. 3-32, 1976.

[49] D. C. Langreth, "Friedel sum rule for Anderson's model of localized impurity states," Physical Review, vol. 150, no. 2, pp. 516-518, 1966.

[50] V. Moldoveanu, H. D. Cornean, and C.-A. Pillet, "Nonequilibrium steady states for interacting open systems: exact results," Physical Review B, vol. 84, no. 7, Article ID 075464, 2011. 

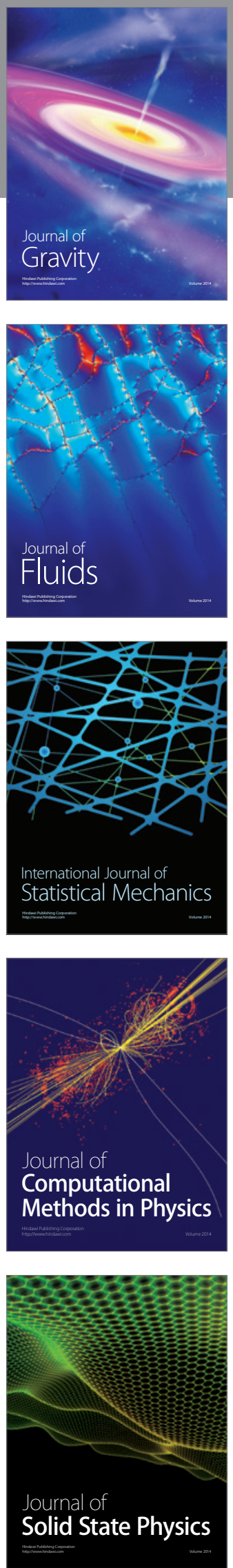

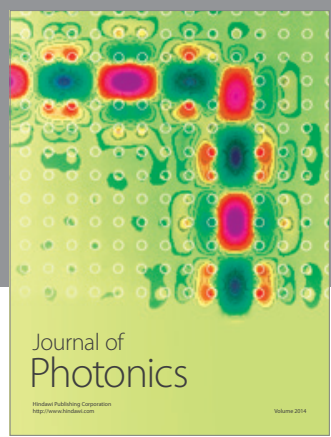

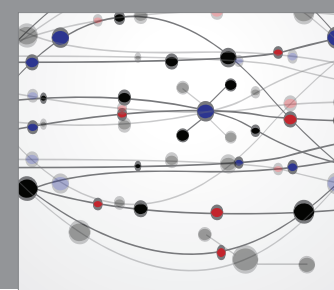

The Scientific World Journal

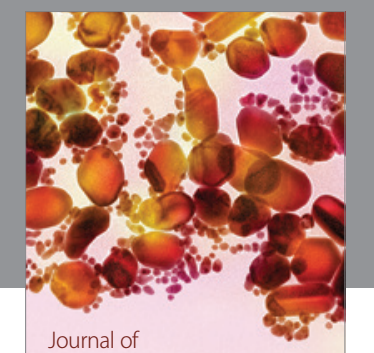

Soft Matter
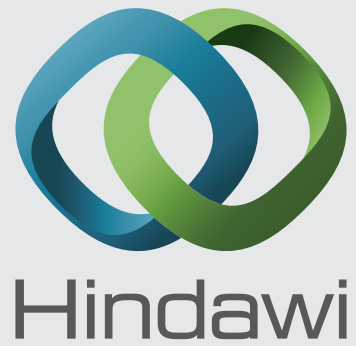

Submit your manuscripts at

http://www.hindawi.com
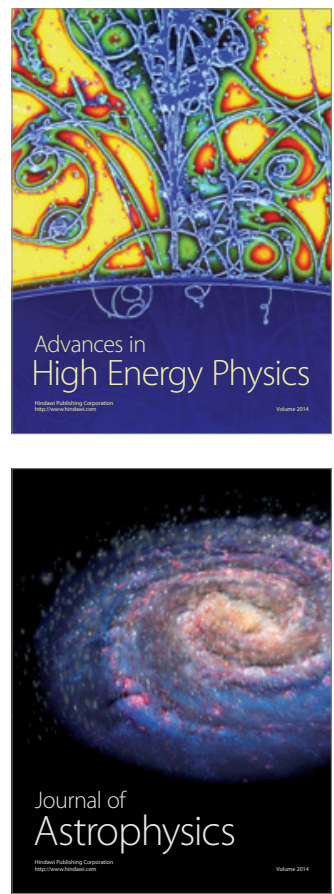
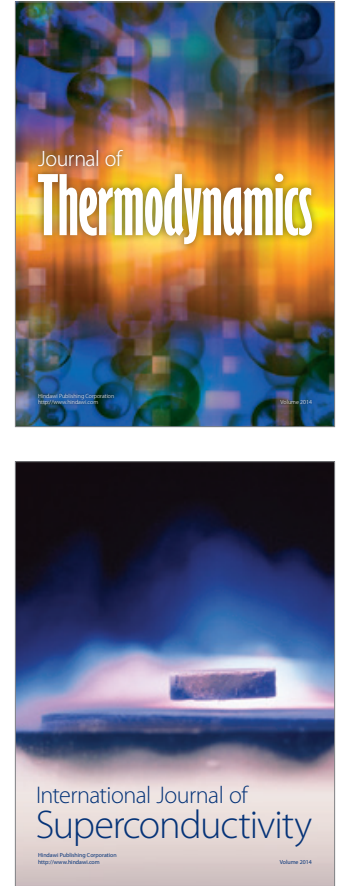
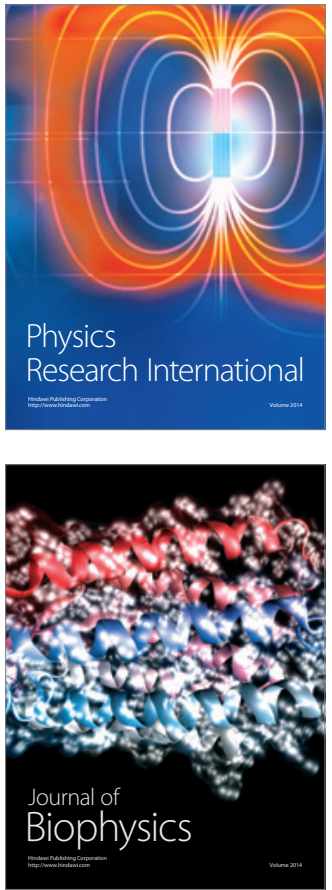
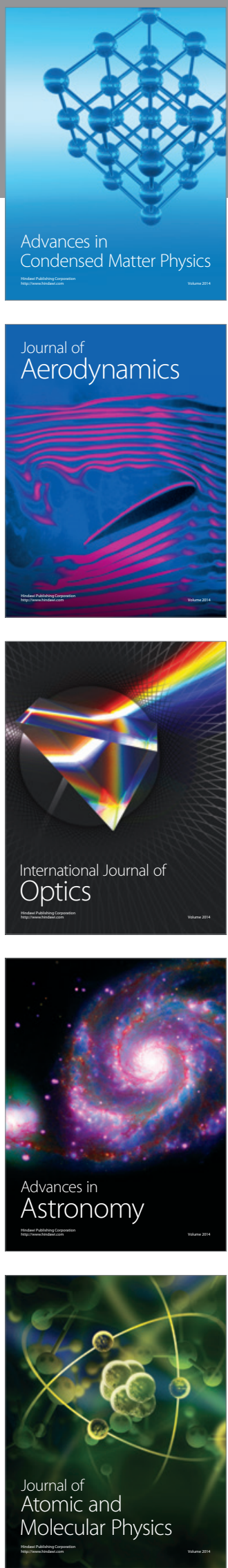